\title{
Epilithic diatoms (Bacillariophyceae) from running waters in NW Iberian Peninsula (Galicia, Spain)
}

\author{
Manel Leira ${ }^{1, *}$, María del Carmen López-Rodríguez ${ }^{2} \&$ Rafael Carballeira ${ }^{3}$ \\ ${ }^{1}$ Laboratório associado IDL, Faculdade de Ciencias, Universidade de Lisboa, 1749-016 Lisbon, Portugal \\ ${ }^{2,3}$ Department of Botany, Biology Faculty, University of Santiago de Compostela, 15076 Santiago de Compostela, Spain \\ ${ }^{1,{ }^{*}}$ Corresponding author: mleira@,fc.ul.pt, http://orcid.org/0000-0001-9024-9448 \\ ${ }^{2}$ mdelcarmen.lopez.rodriguez@usc.es, http://orcid.org/0000-0001-5814-1066 \\ ${ }^{3}$ rafael.carballeira@usc.es, http://orcid.org/0000-0002-2807-6942
}

\begin{abstract}
A catalog of Bacillariophyceae for the rivers of NW of Spain is made for the first time. It includes a re-examination of the taxa reported in previous publications, taxa cited during the last years of the rivers of the Galicia-Costa Hydrographic Demarcation, and a revision of the taxonomy, in line with the systematic and nomenclature changes, which have occurred mainly in recent years. The epilethic diatoms of the river basins of Galicia-Costa were sampled during the years 2005, 2006, and 2007, in May-June and September. The study was carried out in 41 localities distributed along 31 rivers, samples were taken in upstream and downstream sections. Diatom communities were compared in all upstream sections of these rivers draining from siliceous substrates. We identified 141 taxa of diatoms from the coast of Galicia. In this paper we present 15 new citations for Galicia, two for Spain and three for the Iberian Peninsula, in addition to a new species recently described. The dominant taxa are: Achnanthidium minutissimum, Achnanthes subhudsonis, Karayevia oblongella, Cocconeis placentula var. euglypta, Gomphonema rhombicum, and Navicula minima. Achnanthes subhudsonis was the most abundant species during the spring and summer months. The remaining species showed no relevant changes regardless of the time of year The results indicate that the river diatoms of Galicia are an important component of the diversity of the ecosystem.
\end{abstract}

Keywords. Bacillariophyceae, diatoms, Galicia, rivers, Spain, streams.
Resumen. Se realiza por primera vez un catálogo de Bacillariophyceae para los ríos del NW de España. Se incluyen un nuevo examen de los táxones recogidos en publicaciones anteriores, datos de táxones citados durante los últimos años de los ríos de la demarcación hidrográfica de Galicia-Costa y una revisión de la taxonomía, en consonancia con los cambios sistemáticos y nomenclaturales, que se han producido sobre todo durante los últimos años. Se estudiaron las diatomeas epilíticas de las cuencas de los ríos de Galicia-Costa entre los años 2005, 2006 y 2007, durante dos temporadas diferentes: mayojunio y septiembre. El estudio se llevó a cabo en 41 localidades distribuidas a lo largo de 31 ríos, donde se tomaron muestras en tramos aguas arriba y aguas abajo. Las comunidades de diatomeas de estos ríos que drenan de sustratos silíceos fueron comparadas en todos los tramos de aguas arriba. Se identificaron 141 táxones de diatomeas de la costa de Galicia. En este trabajo presentamos 15 nuevas citas para Galicia, dos para España y tres para la Península Ibérica, además de una nueva especie recientemente descrita. Los táxones dominantes son: Achnanthidium minutissimum, Achnanthes subhudsonis, Karayevia oblongella, Cocconeis placentula var. euglypta, Gomphonema rhombicum, y Navicula minima. Achnanthes subhudsonis fue la especie más abundante durante los meses de primavera y verano. Las especies restantes no mostraron cambios pertinentes independientemente de la época del año. Los resultados indican que las diatomeas de los ríos de Galicia son un componente importante de la diversidad del ecosistema.

Palabras clave. Arroyos, Bacillariophyceae, diatomeas, España, Galicia, ríos.

Leira M., López-Rodríguez M.C. \& Carballeira R. 2017. Epilithic diatoms (Bacillariophyceae) from running waters in NW Iberian Peninsula (Galicia, Spain). Anales Jard. Bot. Madrid 74 (2): e062. https://dx.doi.org/10.3989/ajbm.2421

Title in Spanish: Diatomeas epilíticas (Bacillariophyceae) de los cursos de agua corriente del NO de la península ibérica (Galicia, España). Received: 19-VI-2015; accepted: 29-I-2016; published online: 1-XII-2017; Associate Editor: A. Flores.

\section{INTRODUCTION}

Despite the fair and extensive floristic and taxonomic knowledge on the European freshwater diatoms, the diatom flora of the Iberian Peninsula is rather poorly known. The freshwater diatoms of Galicia - NW Spainhave never been thoroughly investigated and there are few works related to some selected localities. The first floristic studies on freshwater diatoms in the region date back to the first half of the $20^{\text {th }}$ century (Gamundi 1911) followed, already during the 50's, by those of Margalef $(1955,1956)$. Later, Varela $(1976,1982)$ made a major contribution, described several taxa with newer data, and published the only freshwater diatom catalogue of Galicia to the date (Varela \& al. 1992). The last taxonomic studies of the diatom flora of Galicia have been published as restricted technical reports on water quality studies in lotic water systems (v.gr., Ector 1992; Penalta-Rodríguez \& LópezRodríguez 2006; Delgado \& al. 2010). Recently, a floristic account has been carried out in small upland streams from two zones catalogued as "site of communitarian importance" proposed by the local government in the Habitats Directive — 92/43/EEC — of the Nature 2000 Network (López-Rodríguez \& Penalta-Rodríguez 2007; Penalta-Rodríguez \& López-Rodríguez 2007). Other more ecologically or paleoecologically orientated publications 
include Bao \& al. (2007). However, despite this growing number of diatom studies in this area, the freshwater diatom flora of Galicia remains rather poorly known. Under these circumstances there is still a need of collecting more data on their distribution and ecological preferences.

This paper reports the results of a systematic survey of the diatom assemblages in Galicia - NW Spain-. The paper describes diatoms collected from running waters in rivers and streams of the Galicia-Costa basin. The study has three main goals: 1) to present a floristic catalogue of the freshwater diatoms living in the rivers of the study area; 2) to provide data on their distribution and autoecology; and 3) to give descriptions of unusual taxa.

\section{MATERIAL AND METHODS}

\section{Study Area}

Galicia is located in the NW of Iberian Peninsula, situated in a transitory zone between the Atlantic and Mediterranean regions under an oceanic climate (fig. 1). The geology is dominated by siliceous rocks: granite in the west and metamorphic rocks in the east. The topography of the area consists mainly of granite rocks, where hills alternate with valleys. The mountain ranges are of low altitude — between 800 and 1,000 m a.s.l.—. The Galician relief is complex and clearly characterised by the presence of steep slopes.

One of the key elements of the Galician landscape is the existence of a large and dense river network controlled by climate and tectonic and the morphological configuration. Galicia-Costa has mild winters and cool summers with precipitation exceeding $1,500 \mathrm{~mm}$ per year while well distributed throughout the year. As a result most of the Galician rivers have an Atlantic or oceanic climate characterized by an abundant and regular flow, with high waters in winter and a moderate drought in the summer. The mountainous geomorphology and regular precipitation influences the occurrence and permanence of many small and medium-sized rivers with regular discharge throughout the year.

The river systems occurring within the area of GaliciaCosta include all Galician river basins that flow into the Bay of Biscay and to the Atlantic Ocean. The rivers of the Bay of Biscay slope flow to the north. These rivers are short with steep gradients. These rivers undergo an oceanic climate with a reduced variation in annual temperature and rain patterns. The Atlantic slope comprises all the rivers flowing west to the Atlantic Ocean. These rivers show a higher environmental and spatial variability, with two groups easily differentiated: the rivers of the Arco ÁrtabroFisterra and the rivers of the Rias Baixas. The proximity

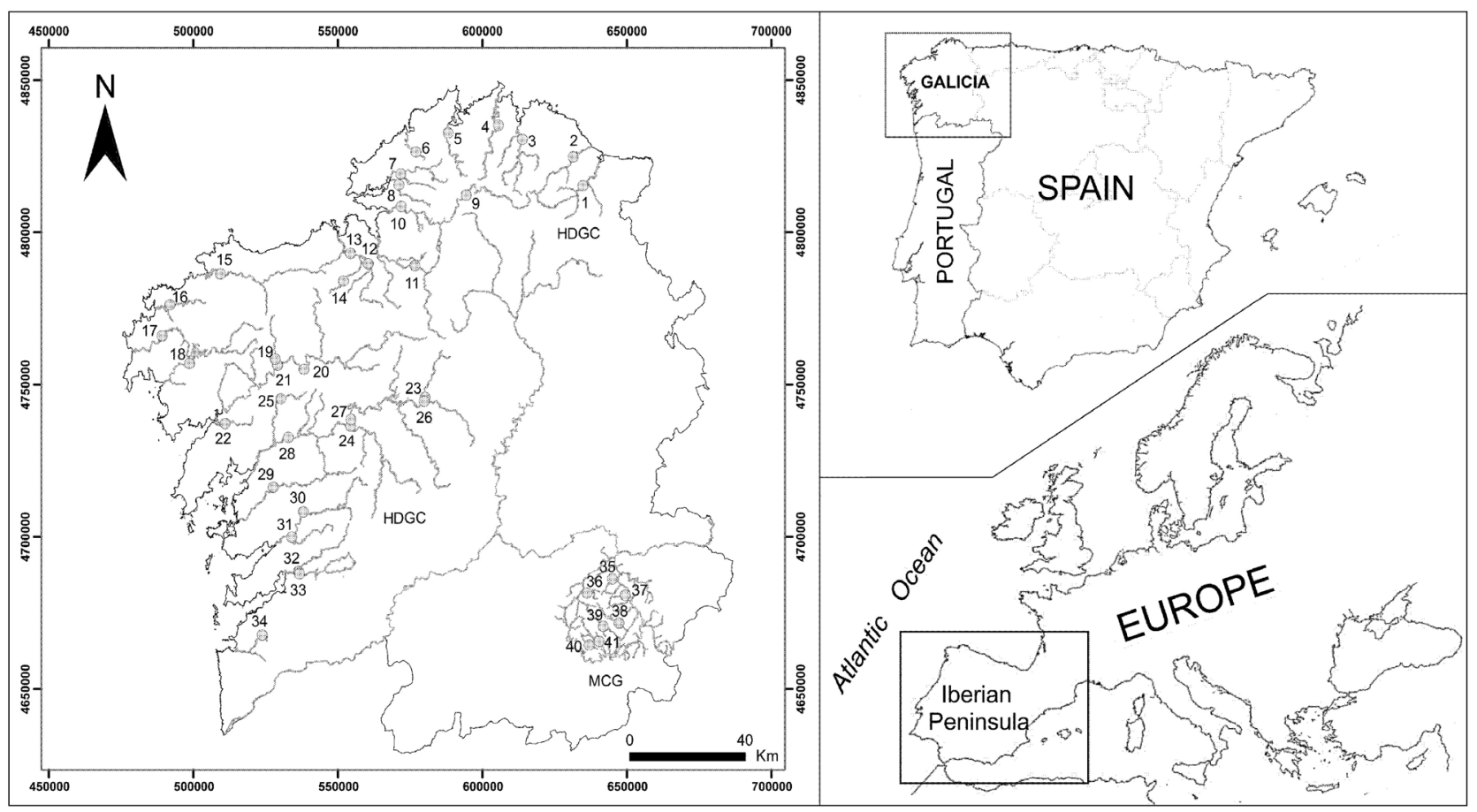

Fig. 1. Map of Galicia sampling sites studied. Sites numbered from 1 to 34 are those included within the Galicia-Costa basins (HDGC). The Macizo Central Gallego (MCG) localities are numbered from 35 to 41 . 
of the mountains to the coast and the oceanic climate of the basins of the Arco Ártabro-Fisterra make all rivers in this area short and quite fast flowing, although they have a summer drought more pronounced than those of the Bay of Biscay. The Rías Baixas rivers are subject to significant Mediterranean influences and undergo severe droughts during summer months.

\section{Sample Collection}

This study was carried out in 41 localities (fig. 1) distributed along 28 rivers in the Galicia-Costa River District. The study has been completed with the inclusion of seven high altitude sites from the mountain ranges of the Macizo Central Gallego (MCG), located in the SE of the studied area. The rivers have been grouped in the five different geographical areas described above according to their drainage basin.

Temperature, $\mathrm{pH}$, conductivity, and dissolved oxygen were measured in situ in each locality with a HANNA HI 9024C microcomputer pH-meter, a HANNA HI 9033 multi-range conductivity meter and a CRISON oxi 45 oxymeter. Water samples for chemical analyses were collected into polypropylene bottles and transported chilled to the laboratory. Water chemistry analyses were carried out at the Department of Physical Chemistry - USC - and included: temperature $-{ }^{\circ} \mathrm{C}-, \mathrm{pH}$, potassium $-\mathrm{mg} \cdot \mathrm{L}^{-1}-$, magnesium $-\mathrm{mg} \cdot \mathrm{L}^{-1}$ - manganese $-\mathrm{mg} \cdot \mathrm{L}^{-1}$ - , chloride - $\mathrm{mg} \cdot \mathrm{L}^{-1}$ —, sulphate $-\mathrm{mg} \cdot \mathrm{L}^{-1}$ —, ammonium $-\mathrm{mg} \cdot \mathrm{L}^{-1}$ conductivity $-\mu \mathrm{S} \cdot \mathrm{cm}^{-1}$ at $20^{\circ} \mathrm{C}$-, nitrates $-\mathrm{mg} \cdot \mathrm{L}^{-1}$-, nitrites - $\mathrm{mg} \cdot \mathrm{L}^{-1}$-, phosphates $-\mathrm{mg} \cdot \mathrm{L}^{-1}$ —, dissolved oxygen $-\mathrm{mg} \cdot \mathrm{L}^{-1}$-, TOC $\left(\mathrm{mg} \cdot \mathrm{L}^{-1}\right)$, BOD5, suspended solids $-\mathrm{mg} \cdot \mathrm{L}^{-1}$-, turbidity $-\mathrm{NTU}$; nephelometric turbidity units - , and alkalinity $-\mathrm{mEq} \cdot \mathrm{L}^{-1}-$. Standard methods for chemical water analysis were carried out following American Public Health Association (APHA 1998).

Localities were sampled between 2002 and 2007 at different moments of the year but more frequently at the end of spring and summer. Diatoms were collected with a brush, from medium-size stones chosen from well lightened and flowing streams. Samples were preserved in a cool-box in the field, until being carried to the laboratory. Diatoms frustules were cleaned with $30 \% \mathrm{H}_{2} \mathrm{O}_{2}$ in hot during 6-7 h. The cleaned frustules were mounted on glass-slides using Naphrax ${ }^{\circledR}$ (CEN 2003, 2004). Identification of the species was carried using an Olympus BX61 microscope equipped with differential interference contrast - DIC, Nomarski- under $1,000 \times$ magnification. A minimum of 400 valves were counted whenever possible. In most cases diatoms were identified to the species level following standard literature (Krammer \& Lange-Bertalot 1985, 1988, 1991a, 1991b) supplemented by more specific works (Krammer 1997a, 1997b, 2000, 2002, 2003; Lange-
Bertalot \& Metzeltin 1996; Lange-Bertalot 1999, 2001; Lange-Bertalot \& Krammer 1989; Reichardt 1999; LangeBertalot \& al. 2011). Additionally, previous references for the Iberian Peninsula were checked after Aboal \& al. (2003). Information about the global distribution and taxonomic status of species recorded during the survey was taken from AlgaeBase (Guiry \& Guiry 2015).

Weighted averaging regression and calibration were perfomed to calculate $\mathrm{pH}$, conductivity, nitrates and phosphates optima of diatom species using the computer program C2 (Juggins 2014). Estimates of optima were assessed comparing the root mean square of prediction - RMSE — and the bootstrap RMSE.

\section{RESULTS AND DISCUSSION}

Characteristics of studied localities are given in table 1: temperature $-{ }^{\circ} \mathrm{C}-, \mathrm{pH}$, conductivity $-\mu \mathrm{S} \cdot \mathrm{cm}^{-1}-$, dissolved oxygen $-\mathrm{mg} \cdot \mathrm{L}^{-1}$ —, water hardness - $\mathrm{mg}$ $\mathrm{CO}_{3} \mathrm{Ca} \cdot \mathrm{L}^{-1}$ - , and catchment area. The majority of the study sites were situated below $20 \mathrm{~m}$ a.s.1., and eight sites occurred between 200-350 m a.s.l. Water temperature ranged from $13.3{ }^{\circ} \mathrm{C}$ to $22.4{ }^{\circ} \mathrm{C}$ among sites within the same sampling season. On average, water temperature varied between $13-20.1{ }^{\circ} \mathrm{C}$ across sites regardless of their altitude. $\mathrm{pH}$ values varied slightly between 6.2 and 7.6. Ten sites showed slightly acidic conditions with a mean $\mathrm{pH}$ between 6.2 and 6.9. Conductivity values ranged from 35 to $457 \mu \mathrm{S} \cdot \mathrm{cm}^{-1}$, with only two localities with values above $200 \mu \mathrm{S} \cdot \mathrm{cm}^{-1}$. Sites are typically acidic with low alkalinity levels.

Results of the diatom analysis are divided into three parts. First, we provide a narrative on some taxa and their distribution to examine relationships between the occurrences of diatom taxa. Second, we establish a provisional taxon checklist for Galicia freshwater diatoms. Finally we give an account of unusual taxa with distribution restricted mostly to Galicia or northwest Iberia.

Galicia river diatoms are a diverse component of the ecosystem, although very distinctive of the Iberian Peninsula diatom flora. Compared with some other geographically and climatically similar areas in northern Spain, Galician running waters are quite distinctive. The geology in the Galician coastal area is homogeneously siliceous, thus rivers and streams are characteristically softwater. Consequently, diatom taxa characteristic of acidic waters were usually well-represented in Galician rivers (table 2). Taxa belonging to the genus Eunotia together with Navicula angusta, Peronia fibula, and Surirella roba were common components of the diatom community throughout all the studied area, although in low numbers. 
Table 1. Localities and physicochemical values. [C, rivers of the Bay of Biscay; AAF, rivers of the Arco ÁrtabroFisterra; ARB, rivers of the Rias Baixas; MCG, rivers of the Macizo Central Gallego.]

\begin{tabular}{|c|c|c|c|c|c|c|c|c|c|}
\hline CODE & RIVER & LOCALITY & COORDINATES & $\mathrm{T}^{\mathrm{a}}$ & $\mathrm{PH}$ & CONDUCTIVITY & $\mathrm{O}_{2}$ & HARDNESS & DRANAIGE \\
\hline & & & & ${ }^{\circ} \mathrm{C}$ & & $\mu \mathrm{S} / \mathrm{cm}$ & $\mathrm{mg} / \mathrm{L}$ & $\mathrm{mg} \mathrm{CO}_{3} \mathrm{Ca} / \mathrm{L}$ & \\
\hline 1 & MASMA & MASMA & 29T0634761 4815296 & 16.1 & 7.6 & 147.9 & 9.6 & 53.2 & $\mathrm{C}$ \\
\hline 2 & OURO & S ACISCLO & 29T0631412 4824765 & 15.9 & 7.0 & 93.3 & 9.6 & 18.7 & $\mathrm{C}$ \\
\hline 3 & LANDRO & NASEIROS & 29T0613811 4830530 & 15.6 & 6.8 & 69.9 & 10.0 & 11.4 & $\mathrm{C}$ \\
\hline 4 & SOR & COTO RIBEIRAS & 29T0605538 4835129 & 16.3 & 6.6 & 66.8 & 9.9 & 10.4 & $\mathrm{C}$ \\
\hline 5 & MERA & MERA DE ABAIXO & $29 \mathrm{~T} 05881294832636$ & 15.1 & 7.3 & 123.5 & 10.1 & 38.3 & $\mathrm{C}$ \\
\hline 6 & FORCADAS & VILABOA & 29T0576983 4826378 & 19.8 & 7.4 & 109.9 & 9.0 & 28.2 & $\mathrm{C}$ \\
\hline 7 & XUBIA & S. SADURNIÑO & $29 \mathrm{~T} 05737084808503$ & 15.8 & 7.4 & 153.2 & 9.3 & 41.3 & AAF \\
\hline 8 & BELELLE & VILADONELLE & 29T0571126 4815639 & 15.8 & 6.5 & 74.0 & 8.8 & 11.5 & AAF \\
\hline 9 & EUME & RIBADEUME & 29T0591148 4820458 & 16.1 & 6.5 & 46.2 & 9.7 & 8.7 & AAF \\
\hline 10 & EUME & OMBRE & 29T0571870 4808503 & 13.0 & 6.4 & 126.2 & 10.7 & 41.8 & AAF \\
\hline 11 & MANDEO & MUNIFERRAL & 29T0576677 4789040 & 16.6 & 7.0 & 83.0 & 9.9 & 14.9 & $\mathrm{AAF}$ \\
\hline 12 & MERO & MABEGONDO & 29T0560483 4789644 & 16.0 & 7.0 & 112.7 & 9.6 & 25.2 & AAF \\
\hline 13 & MERO & CAMBRE & 29T0554293 4793045 & 20.1 & 7.1 & 195.0 & 9.5 & 58.7 & AAF \\
\hline 14 & BARCÉS & RIBEIRA & 29T05518994784011 & 16.5 & 7.1 & 456.7 & 9.8 & 167.0 & AAF \\
\hline 15 & ANLLONS & ANLLÓNS & $29 \mathrm{~T} 05093584786303$ & 16.9 & 7.3 & 173.1 & 10.0 & 40.8 & AAF \\
\hline 16 & GRANDE & PONTE DO PORTO & 29T0491863 4776196 & 17.2 & 6.7 & 93.4 & 9.7 & 15.8 & $\mathrm{AAF}$ \\
\hline 17 & CASTRO & SENANDE & 29T04891924765965 & 16.6 & 6.3 & 85.7 & 9.6 & 10.8 & $\mathrm{AAF}$ \\
\hline 18 & XALLAS & PTE OLVEIRA & 29T0498653 4756899 & 18.7 & 6.7 & 74.5 & 9.0 & 16.9 & ARB \\
\hline 19 & DUBRA & PORTOMOURO & 29T0529113 4756357 & 14.9 & 7.2 & 90.1 & 10.4 & 26.8 & ARB \\
\hline 20 & TAMBRE & CHAIÁN & 29T0538222 4754950 & 17.0 & 7.0 & 98.3 & 9.9 & 27.3 & ARB \\
\hline 21 & TAMBRE & PORTOMOURO & 29T0528303 4758349 & 17.6 & 7.2 & 98.6 & 10.2 & 23.9 & $\mathrm{ARB}$ \\
\hline 22 & TRABA & $\begin{array}{l}\text { NOIA- } \\
\text { PORTOBRAVO }\end{array}$ & 29T0510919 4737178 & 16.4 & 6.2 & 72.3 & 9.9 & 10.7 & $\mathrm{ARB}$ \\
\hline 23 & $\begin{array}{l}\text { ULLA- } \\
\text { FURELOS }\end{array}$ & PONTE BARAZÓN & 29T0580147 4745726 & 15.7 & 7.3 & 122.1 & 9.0 & 38.4 & ARB \\
\hline 26 & ULLA-DEZA & CIRA & 29T0554401 4736284 & 16.9 & 7.2 & 106.5 & 10.1 & 26.5 & ARB \\
\hline 27 & ULLA-SAR & BERTAMIRÁNS & $29 \mathrm{~T} 05302474745287$ & 17.6 & 7.4 & 310.8 & 8.3 & 55.3 & $\mathrm{ARB}$ \\
\hline 24 & ULLA & $\begin{array}{l}\text { SANTISO } \\
\text { (MOURAZOS) }\end{array}$ & 29T0579849 4744500 & 15.6 & 7.2 & 77.9 & 9.8 & 20.1 & ARB \\
\hline 25 & ULLA & PTE LEDESMA & 29T0554492 4738488 & 16.5 & 7.3 & 93.7 & 9.7 & 27.9 & $\mathrm{ARB}$ \\
\hline 28 & ULLA & CARCACÍA & 29T0532844 4732618 & 18.3 & 7.3 & 93.9 & 9.9 & 25.1 & ARB \\
\hline 29 & UMIA & $\begin{array}{l}\text { COTO DE } \\
\text { CALDAS }\end{array}$ & 29T0527579 4716262 & 18.3 & 6.8 & 55.8 & 8.9 & 9.5 & ARB \\
\hline 31 & LEREZ & CUTIÁN & 29T0538023 4708198 & 17.4 & 6.7 & 40.1 & 9.1 & 7.4 & ARB \\
\hline 30 & LEREZ & BORA & 29T0534017 4699819 & 19.1 & 6.7 & 37.9 & 10.7 & - & ARB \\
\hline 32 & VERDUGO & SOUTOMAIOR & 29T0536405 4688537 & 18.5 & 6.8 & 39.2 & 9.5 & 4.9 & ARB \\
\hline 33 & OITAVEN & SOUTOMAIOR & 29T0536676 4687826 & 17.1 & 6.5 & 35.0 & 9.6 & 5.0 & $\mathrm{ARB}$ \\
\hline 34 & ZAMÁNS & ZAMÁNS & 29T0523794 4667710 & 19.7 & 6.9 & 61.7 & 9.1 & 11.3 & $\mathrm{ARB}$ \\
\hline 35 & \multicolumn{2}{|l|}{ SAN LÁZARO } & 29T644962 4686175 & 6.3 & 7.5 & 60.0 & - & - & MCG \\
\hline 36 & \multicolumn{2}{|c|}{ TORNOS-PALLEIRAS } & 29T636432 4683447 & 12.7 & 6.6 & 12.7 & 88.5 & - & MCG \\
\hline 37 & \multicolumn{2}{|c|}{ CANEIRO-REQUEIX0 } & 29T647398 4687699 & 18.6 & 6.8 & 89.3 & 9.7 & - & MCG \\
\hline 38 & \multicolumn{2}{|l|}{ CENZA } & 29T647250 4671662 & 24.5 & 7.1 & 20.2 & 79.1 & - & $\mathrm{MCG}$ \\
\hline 39 & \multicolumn{2}{|c|}{ PRADOALBAR-CONSO } & 29T641788 4670533 & 16.7 & 6.9 & 15.0 & 9.8 & - & MCG \\
\hline 40 & \multicolumn{2}{|c|}{ INVERNADEIRO SUR } & 29T636900 4664375 & 17.5 & 7.0 & 128.3 & 9.1 & - & MCG \\
\hline 41 & \multicolumn{2}{|c|}{ INVERNADEIRO NORTE } & 29T640350 4665600 & 18.4 & 6.7 & 43.3 & 9.3 & - & MCG \\
\hline
\end{tabular}


Table 2. Optima calculated for the most common and abundant diatoms. Values were calculated using Weighted Averages as implemented in C2 (Juggins 2014).

\begin{tabular}{|c|c|c|c|c|}
\hline & $\mathrm{pH}$ & Conductivity & Nitrates & Phosphates \\
\hline & & $\mu \mathrm{S} \mathrm{cm}^{-1}$ & $\mathrm{mg} \mathrm{L} \mathrm{L}^{-1}$ & $\mathrm{mg} \mathrm{L} \mathrm{L}^{-1}$ \\
\hline Achnanthes oblongella Østrup & 6.7 & 72.3 & 1.519 & 0.041 \\
\hline Achnanthidium chlidanos (Hohn \& Hellerman) Novelo, Tavera \& Ibarra & 6.1 & 43.5 & 1.338 & 0.024 \\
\hline Achnanthidium lanceolatum Bréb. ex Kütz. & 6.9 & 78.1 & 2.887 & 0.113 \\
\hline Achnanthidium minutissimum Kütz. & 6.7 & 55.1 & 1.252 & 0.026 \\
\hline Achnanthidium subatomoides (Hust.) Monnier, Lange-Bert. \& Ector & 6.6 & 43.3 & 0.837 & 0.020 \\
\hline Adlafia bryophila (J.B.Petersen) Moser, Lange-Bert. \& Metzeltin & 6.8 & 45.7 & 0.826 & 0.054 \\
\hline Achnanthidium subhudsonis (Hust.) H.Kobayasi & 7.0 & 78.9 & 1.373 & 0.059 \\
\hline Cocconeis placentula var. euglypta (Ehrenb.) Grunow & 7.0 & 79.1 & 1.442 & 0.044 \\
\hline Cymbella aequalis $\mathrm{W} . \mathrm{Sm}$. & 6.1 & 48.2 & 0.487 & 0.019 \\
\hline Cymbella aspera (Ehrenb.) Cleve & 6.7 & 81.3 & 0.503 & 0.032 \\
\hline Diatoma mesodon (Ehrenb.) Kütz. & 6.7 & 35.1 & 1.885 & 0.017 \\
\hline Encyonema minutum (Hilse) D.G.Mann & 7.1 & 67.1 & 2.522 & 0.059 \\
\hline Encyonema silesiacum (Bleisch) D.G.Mann & 6.8 & 50.0 & 1.377 & 0.027 \\
\hline Eunotia bilunaris var. bilunaris (Ehrenb.) Mills & 6.3 & 38.7 & 0.538 & 0.003 \\
\hline Eunotia exigua (Bréb. ex Kütz.) Rabenh. & 6.3 & 38.5 & 0.941 & 0.022 \\
\hline Eunotia faba Grunow & 6.5 & 31.9 & 0.384 & 0.003 \\
\hline Eunotia implicata Nörpel, Lange-Bert. \& Alles & 6.3 & 51.1 & 0.824 & 0.026 \\
\hline Eunotia incisa W.Greg. & 6.3 & 31.8 & 0.284 & 0.004 \\
\hline Eunotia intermedia (Krasske ex Hust.) Nörpel \& Lange-Bert. & 6.2 & 35.1 & 0.847 & 0.011 \\
\hline Eunotia minor (Kütz.) Grunow & 6.3 & 38.2 & 1.065 & 0.030 \\
\hline Eunotia paludosa (W.Sm.) Reimer var. paludosa & 6.1 & 34.0 & 0.842 & 0.005 \\
\hline Eunotia pectinalis (Kütz.) Rabenh. var. undulata (Ralfs) Rabenh. & 6.4 & 51.2 & 1.016 & 0.015 \\
\hline Eunotia praerupta Ehrenb. & 6.6 & 36.0 & 1.497 & 0.007 \\
\hline Eunotia subarcuatoides Alles, Nörpel \& Lange-Bert. & 6.1 & 38.6 & 0.842 & 0.021 \\
\hline Fragilaria capucina Desm. var. capucina & 6.6 & 42.7 & 0.528 & 0.006 \\
\hline Fragilaria capucina var. vaucheriae (Kütz.) Lange-Bert. & 6.8 & 51.5 & 2.938 & 0.035 \\
\hline Fragilaria capucina subsp. rumpens (Kütz.) Lange-Bert. & 6.6 & 55.9 & 1.176 & 0.017 \\
\hline Fragilaria pinnata Ehrenb. var. pinnata & 7.0 & 70.5 & 1.554 & 0.056 \\
\hline Fragilaria virescens Ralfs & 6.4 & 41.9 & 1.395 & 0.039 \\
\hline Frustulia saxonica Rabenh. & 6.5 & 60.9 & 2.520 & 0.119 \\
\hline Frustulia vulgaris (Thwaites) De Toni & 6.6 & 59.3 & 3.374 & 0.072 \\
\hline Gomphonema exilissimum (Grunow) Lange-Bert. \& E.Reichardt & 6.5 & 45.5 & 0.960 & 0.048 \\
\hline Gomphonema gracile Ehrenb. & 6.5 & 44.1 & 0.691 & 0.030 \\
\hline Gomphonema minutum (C.Agardh) C.Agardh f. minutum & 6.9 & 75.5 & 2.118 & 0.120 \\
\hline Gomphonema parvulum (Kütz.) Kütz. var. parvulum f. parvulum & 6.7 & 68.8 & 1.693 & 0.089 \\
\hline Gomphonema rhombicum Fricke & 6.9 & 52.9 & 1.724 & 0.020 \\
\hline $\begin{array}{l}\text { Humidophila contenta (Grunow) Lowe, Kociolek, J.R.Johans., Van de Vijver, Lange-Bert. \& } \\
\text { Kopalová }\end{array}$ & 6.8 & 49.0 & 1.214 & 0.038 \\
\hline Iconella delicatissima (F.W.Lewis) Ruck \& Nakov & 6.0 & 44.0 & 0.761 & 0.002 \\
\hline Navicula angusta Grunow & 6.4 & 39.9 & 0.637 & 0.010 \\
\hline Navicula cryptocephala Kütz. & 6.7 & 60.8 & 1.450 & 0.070 \\
\hline Navicula cryptotenella Lange-Bert. & 7.0 & 75.2 & 1.277 & 0.042 \\
\hline Navicula exilis Kütz. & 6.7 & 67.3 & 2.113 & 0.149 \\
\hline Navicula gregaria Donkin & 7.0 & 71.1 & 3.885 & 0.093 \\
\hline Navicula lanceolata (C.Agardh) Ehrenb. & 6.9 & 82.8 & 3.146 & 0.085 \\
\hline Navicula minima Grunow & 6.9 & 115.0 & 3.436 & 0.381 \\
\hline Navicula rynchocephala Kütz. & 6.8 & 66.3 & 1.433 & 0.060 \\
\hline Nitzschia dissipata (Kütz.) Grunow var. dissipata & 6.8 & 54.5 & 2.111 & 0.046 \\
\hline Nitzschia palea (Kütz.) W.Sm. & 6.9 & 86.2 & 2.404 & 0.266 \\
\hline Nitzschia palea var. debilis (Kütz.) Grunow & 6.5 & 51.6 & 1.273 & 0.010 \\
\hline Pinnularia divergens W.Sm. var. divergens & 6.8 & 92.1 & 0.283 & 0.046 \\
\hline Pinnularia subcapitata W.Greg. var. subcapitata & 6.3 & 38.8 & 1.391 & 0.044 \\
\hline Planothidium daui (Foged) Lange-Bert. & 6.7 & 59.0 & 0.734 & 0.009 \\
\hline Reimeria sinuata (W.Greg.) Kociolek \& Stoermer & 6.9 & 62.0 & 1.633 & 0.024 \\
\hline Surirella roba Leclercq & 6.1 & 38.7 & 1.154 & 0.007 \\
\hline Tabellaria flocculosa (Roth) Kütz. & 6.6 & 64.3 & 1.735 & 0.014 \\
\hline
\end{tabular}


Diatom communities showed a high degree of similarity among sites and were comparable in all upstream stretches of these rivers draining siliceous substrates. Dominant epilithic diatom taxa were Achnanthidium minutissimum, Achnanthes subhudsonis, Karayevia oblongella, Cocconeis placentula var. euglypta, Gomphonema rhombicum, and Navicula minima. Achnanthes subhudsonis was the most abundant species during the spring and summer months. The remaining species showed no relevant changes regardless of the time of year. Other species which also occurred frequently but were less abundant were: Amphora pediculus, Cocconeis placentula var. pseudolineata, Encyonema minutum, Fragilaria capucina subsp. rumpens, Gomphonema parvulum, Melosira varians, Navicula angusta, Navicula cryptocephala, Navicula cryptotenella, Navicula lanceolata, Navicula minima, Navicula rhyncocephala, Nitzschia dissipata, Nitzschia palea, Planothidium lanceolatum, Psammothidium subatomoides, Ulnaria ulna, and Ulnaria biceps.

Overall, 141 taxa have been found which can be classified in 48 genera and six families. The taxa that are new records for Iberian Peninsula are marked with three asterisk $(* * *)$, for Spain with two asterisk $(* *)$, the records which are new to Galicia with an asterisk (*). Locality numbers for the new records and figures are indicated after each taxon within brackets.

\section{Coscinodiscophyceae}

$$
\begin{aligned}
& \text { Aulacoseira alpigena (Grunow) Krammer (fig. 2b)* } \\
& \text { Aulacoseira ambigua (Grunow) Simonsen (fig. 2a)* } \\
& \text { Aulacoseira distans (Ehrenb.) Simonsen (fig. 2c) } \\
& \text { Cyclotella meneghiniana Kütz. (fig. 2e) } \\
& \text { Discostella stelligera (Cleve \& Grunow) Houk \& Klee (fig. 2f) } \\
& \text { Melosira varians C.Agardh (fig. 2d) }
\end{aligned}
$$

\section{Fragilariophyceae}

$$
\text { Asterionella formosa Hassall (fig. 2r) }
$$$$
\text { Diatoma mesodon (Ehrenb.) Kütz. (fig. 2s) }
$$$$
\text { Diatoma vulgaris Bory (fig. 2t) }
$$$$
\text { Fragilaria bidens Heib. (fig. 2j) }
$$

Fragilaria capucina var. capitellata (Grunow) Lange-Bert. (fig. 2g)

Fragilaria capucina var. capucina Desm. (fig. 2i)

Fragilaria capucina var. rumpens (Kutz.) Lange-Bert. (fig. 2h)

Fragilaria capucina var. vaucheriae (Kütz.) Lange-Bert. (fig. 2m)

Fragilaria crotonensis Kitton (fig. 2q)

Fragilaria parasitica (W.Sm.) Grunow (fig. 2k) *

Fragilariforma virescens (Ralfs) D.M. Williams \& Round (fig. 21)

Hannaea arcus (Ehrenb.) R.M.Patrick (fig. 2n)

Meridion circulare var. constrictum (Ralfs) Van Heurck (fig. 2v)
Tabellaria floculosa (Roth.) Kütz. (fig. 2u)

Ulnaria biceps (Kütz.) Compère (fig. 20)*

Ulnaria ulna (Nitzsch) Compère (fig. 2p)

\section{Bacilariophyceae}

Achnantes brevipes var. intermedia (Kütz.) Cleve (fig. 4o)*

Achnanthes subhudsonis Hust. (fig. 41)

Achnanthidium minutissimum (Kütz.) Czarneki (fig. 4m)

Achnanthidium subatomus (Hust.) Lange-Bert. (fig. 4n)

Amphora inaeriensis Krammer (fig. 9n)*

Amphora ovalis (Kütz.) Kütz. (fig. 9m)

Amphora pediculus (Kütz.) Grunow (fig. 9o)

Bacillaria paradoxa J.F.Gmel. (fig. 10a)*

Brachysira intermedia (Østrup) Lange-Bert. (fig. 5a)

Brachysira neglectissima Lange-Bert. (fig. 5c)

Brachysira neoexilis Lange-Bert. (fig. 5b)

Caloneis bacillum (Grunow) Cleve (fig. 6b)

Caloneis molaris (Grunow) Krammer (fig. 6c)

Caloneis silicula (Ehrenb.) Cleve (fig. 6d)

Cavinula variostriata (Krasske) D.G.Mann \& Stickle (fig. 5d) *

Cocconeis pediculus Ehrenb. (fig. 4r)

Cocconeis placentula var. euglypta (Ehrenb.) Grunow (fig. 4p)

Cocconeis placentula var. lineata (Ehrenb.) Van Heurck (fig. 4q)

Cocconeis placentula var. pseudolineata Geitler (fig. 4s)

Craticula buderi (Hust.) D.G.Mann (fig. 8 h)*

Cymbella aspera (Ehrenb.) Cleve (fig. 8 k)

Cymbella tumida (Bréb.) Van Heurck (fig. 8 j)

Diploneis elliptica (Kütz.) Cleve (fig. 5n)

Diploneis oculata (Bréb.) Cleve (fig. 5o)

Diploneis ovalis (Hilse) Cleve (fig. $5 \mathrm{~m}$ )

Encyonema gracile Rabenh. (fig. $8 \mathrm{~m}$ )

Encyonema minutum (Hilse) D.G.Mann (fig. 8i)

Encyonema silesiacum (Bleisch) D.G.Mann (fig. 81)

Eunotia bilunaris (Ehrenb.) Mills (fig. 3a)

Eunotia exigua (Breb. ex Kütz.) Rabenh. (fig. 3e)

Eunotia faba Ehrenb. (fig. 3c)

Eunotia formica Ehrenb. (fig. 3b)

Eunotia implicata Nörpel, Lange-Bert. \& Alles (fig. 3f)

Eunotia incisa W.Greg. (fig. 3g)

Eunotia intermedia (Krasske ex Hust.) Nörpel \& Lange-Bert. (fig. 3j)

Eunotia minor (Kütz.) Grunow (fig. 3i)

Eunotia pectinalis var. undulata (Ralfs) Rabenh. (fig. 3k)

Eunotia praerupta Ehrenb. (fig. 31)

Eunotia soleirolli (Kutz.) Rabenh. (fig. 3m)* 


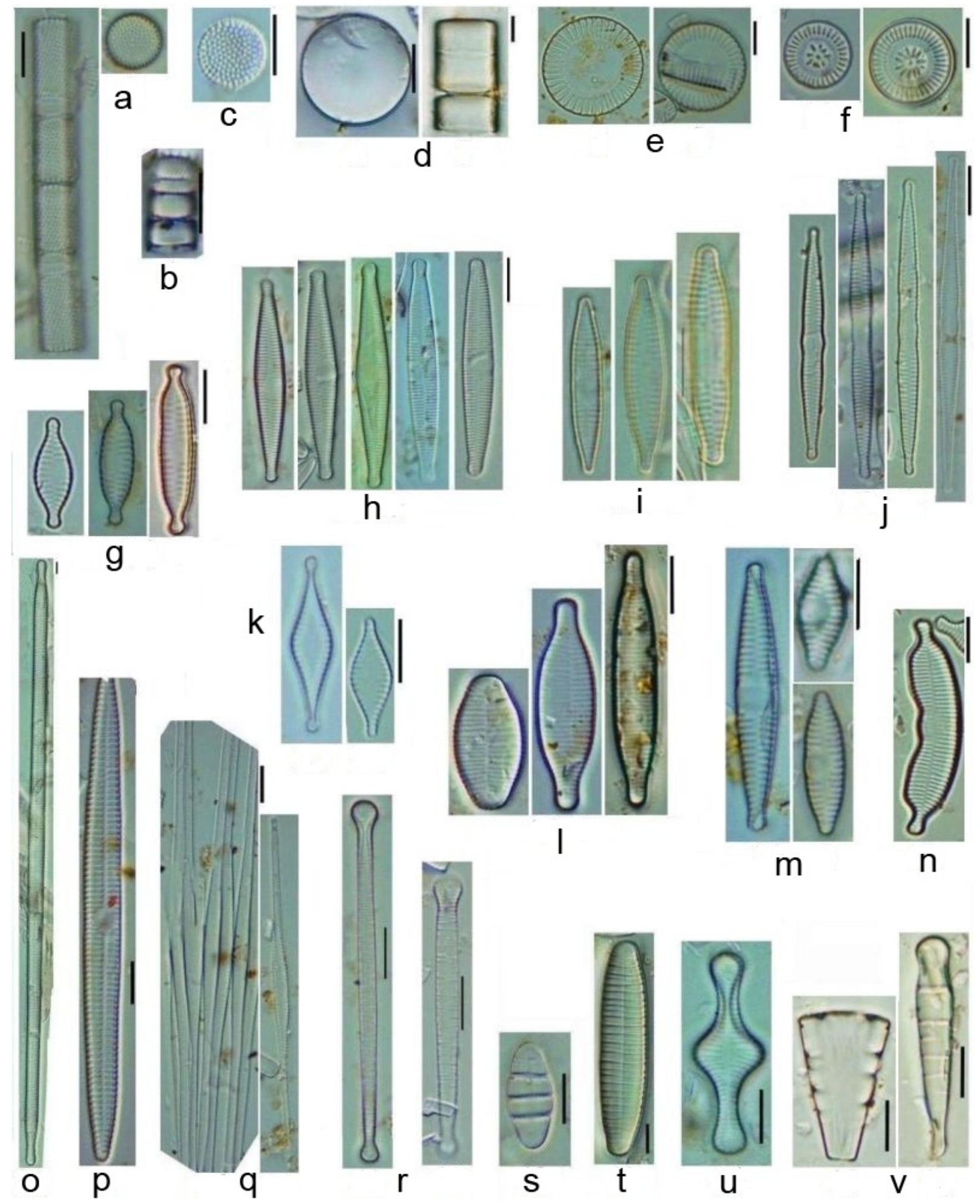

Fig. 2. Epilithic diatoms (Coscinodiscophyceae-Fragilariophyceae): a, Aulacoseira ambigua; b, Aulacoseira alpigena; c, Aulacoseira distans; d, Melosira varians; e, Cyclotella meneghiniana; f, Discostella stelligera; g, Fragilaria capucina var. capitellata; h, Fragilaria capucina var. rumpens; i, Fragilaria capucina var. capucina; j, Fragilaria bidens; k, Fragilaria parasitica; l, Fragilariaforma virescens; m, Fragilaria capucina var. vaucheriae; n, Hannaea arcus; o, Ulnaria biceps; p, Ulnaria ulna; q, Fragilaria crotonensis; r, Asterionella formosa; s, Diatoma mesodon; t, Diatoma vulgaris; u, Tabellaria floculosa; v, Meridion circulare var. constrictum. [Scale bars $6 \mu \mathrm{m}$.] 

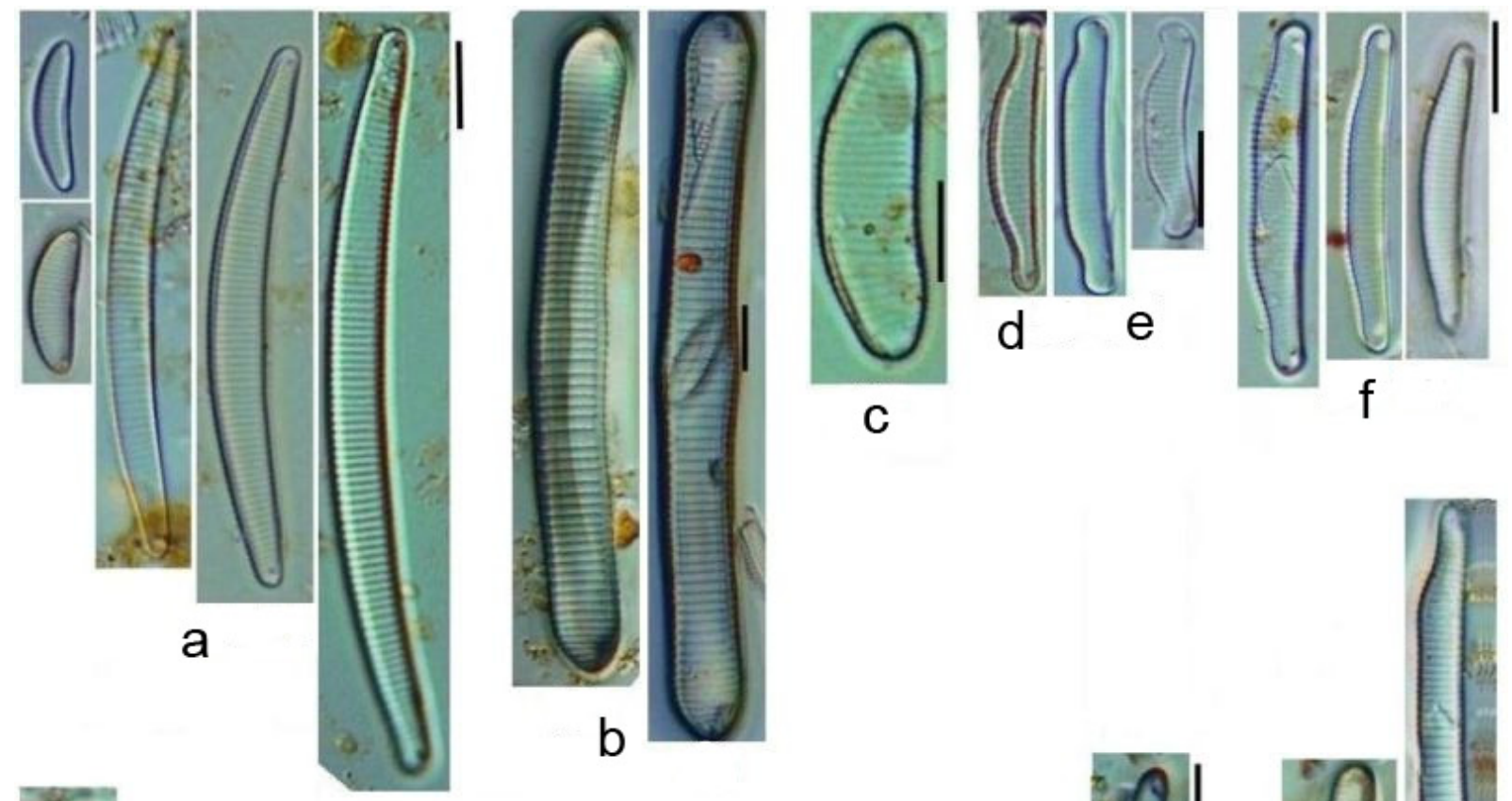

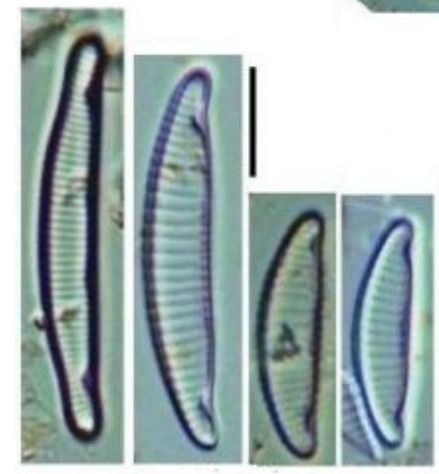

g
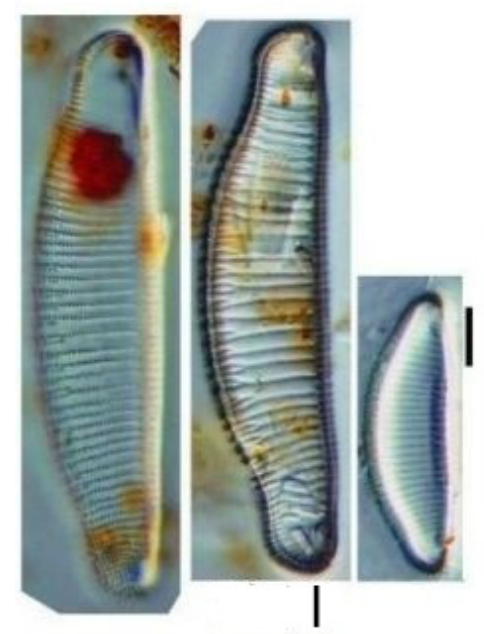

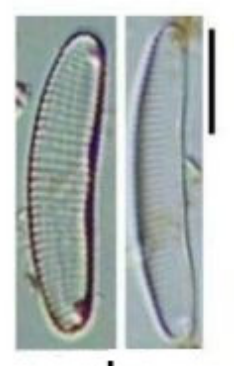

$\mathrm{h}$

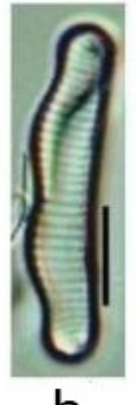

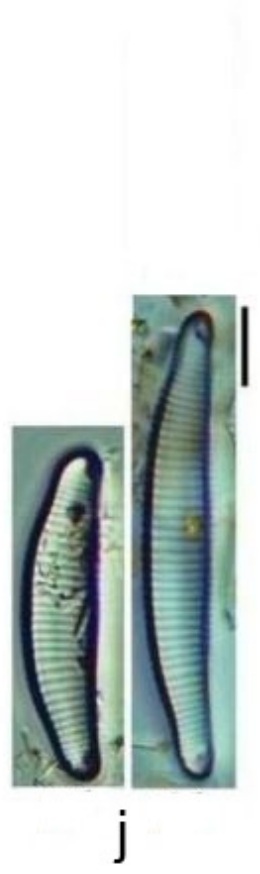
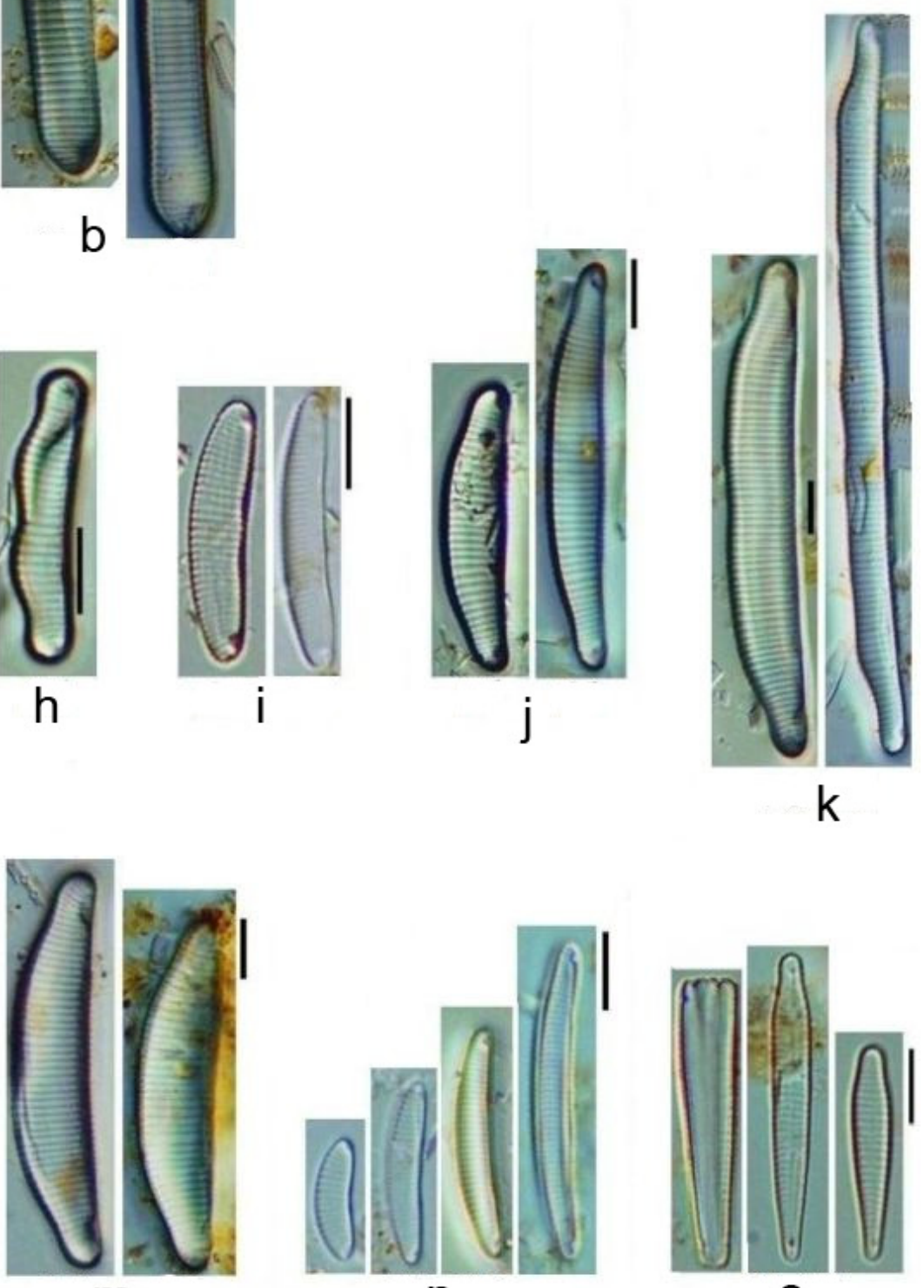

$\mathrm{m}$

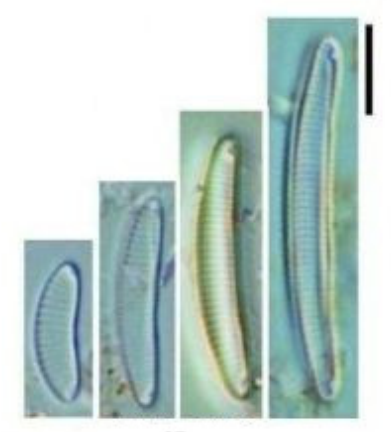

$\mathrm{n}$

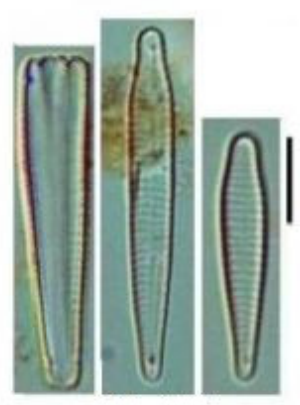

$\mathrm{O}$

Fig. 3. Epilithic diatoms (Bacillariophyceae): a, Eunotia bilunaris; b, Eunotia formica; c, Eunotia faba; d, Eunotia sp.; e, Eunotia exigua; f, Eunotia implicata; $\mathbf{g}$, Eunotia incisa; $\mathbf{h}$, Eunotia islandica; i, Eunotia minor; j, Eunotia intermedia; k, Eunotia pectinalis var. undulata; $\mathbf{l}$, Eunotia praerupta; m, Eunotia soleirolii; n, Eunotia subarcuatoides; o, Peronia erinacea. [Scale bars $6 \mu \mathrm{m}$.] 


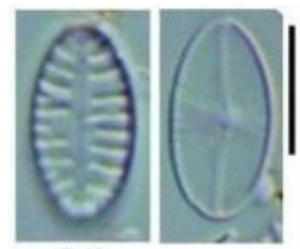

a

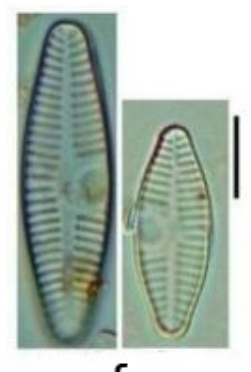

f

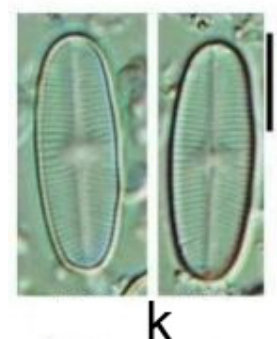

k
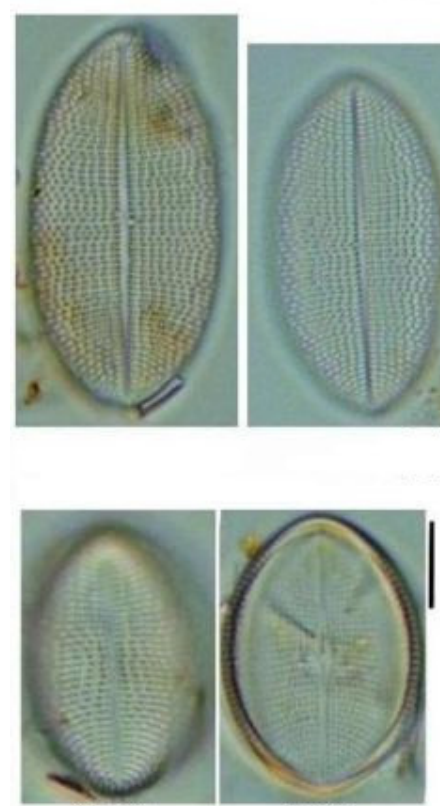

r

g

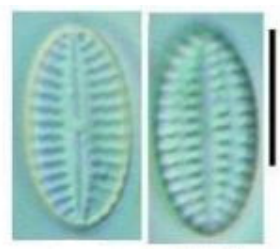

b
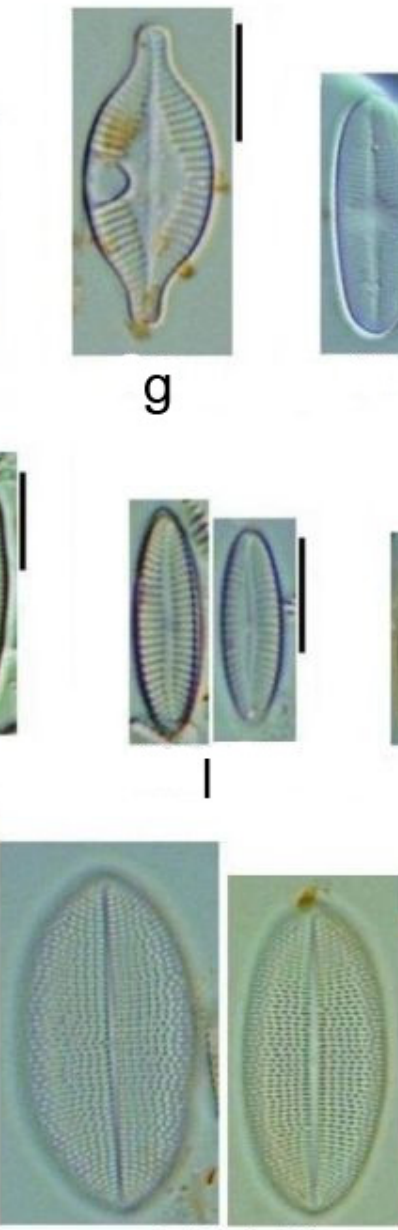

$\mathrm{p}$

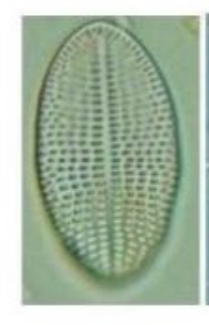

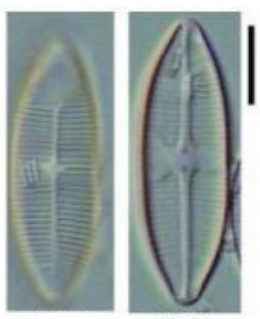

C

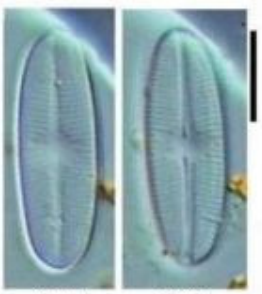

h
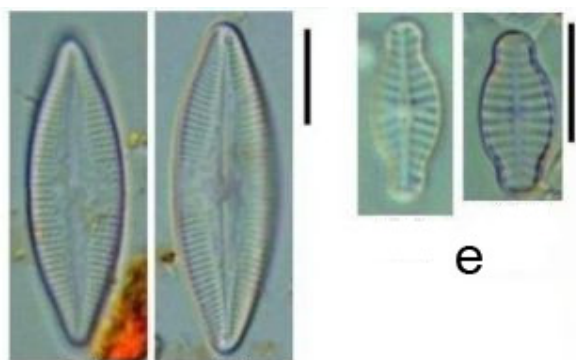

e

\section{d}

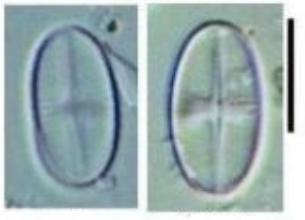

i

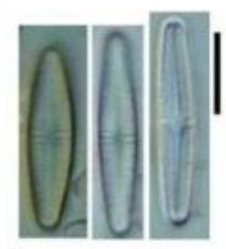

m

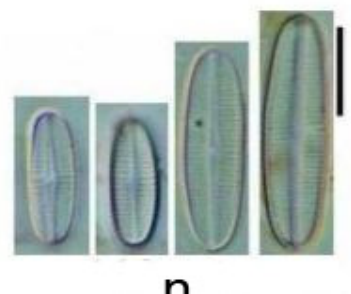

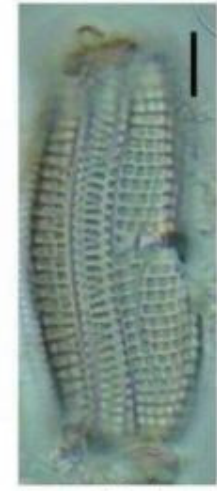

0

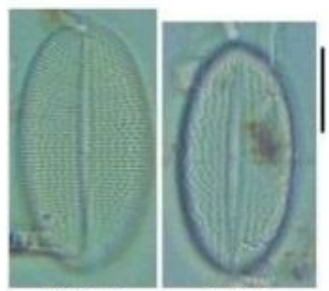

q

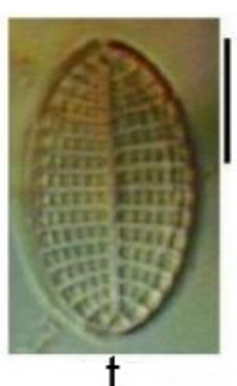

Fig. 4. Epilithic diatoms (Bacillariophyceae): a, Karayevia oblongella; b, Kolbesia suchlandtii; c, Lemnicola hungarica; d, Nupela lapidosa; e, Planothidium daui; $\mathbf{f}$, Planothidium lanceolatum; $\mathbf{g}$, Planothidium peragalli; $\mathbf{h}$, Psammothidium helveticum; i, Psammothidium subatomoides; $\mathbf{j}$, Achnanthes scotica; $\mathbf{k}$, Achnanthes chlidanos; $\mathbf{l}$, Achnanthes subhudsonis; m, Achnanthidium minutissimum; n, Achnanthidium subatomus; $\mathbf{0}$, Achnanthes brevipes var. intermedia; $\mathbf{p}$, Cocconeis placentula var. euglypta; q, Cocconeis placentula var. lineata; r, Cocconeis pediculus; s, Cocconeis placentula var. pseudolineata; $\mathbf{t}$, Cocconeis scutellum. [Scale bars $6 \mu \mathrm{m}$.] 


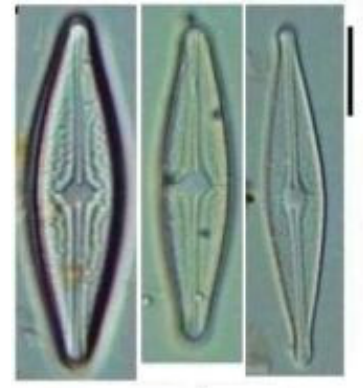

a

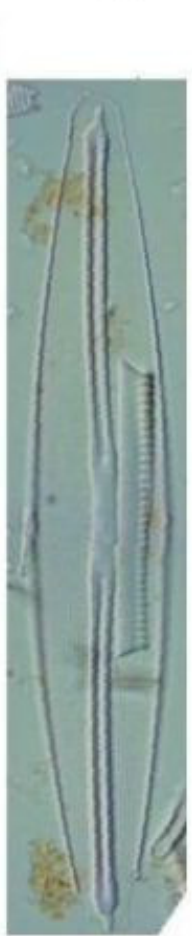

$\mathrm{h}$

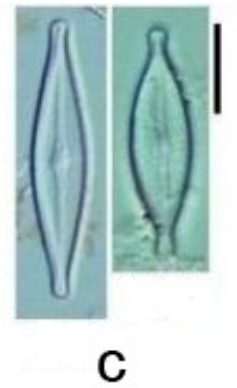

b

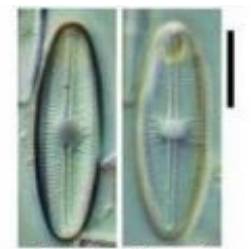

d

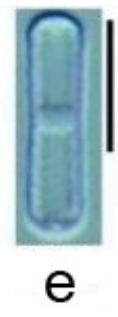

e
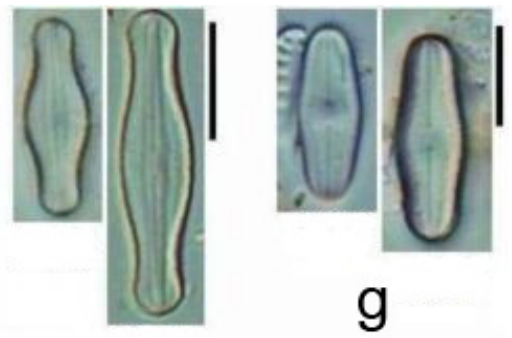

g
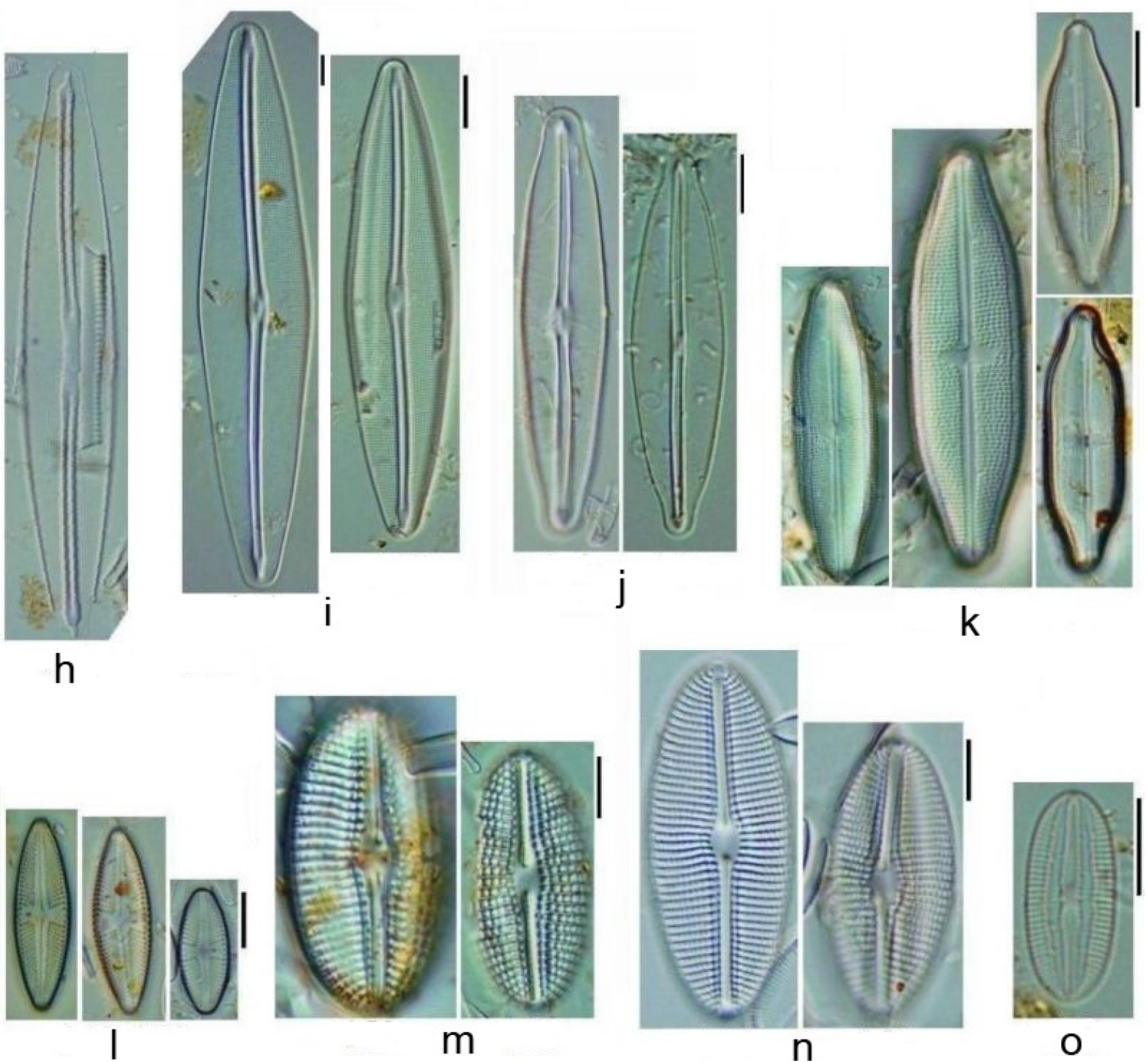

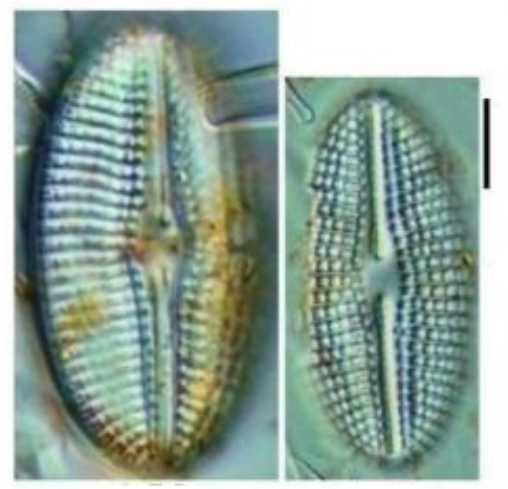

$\mathrm{m}$

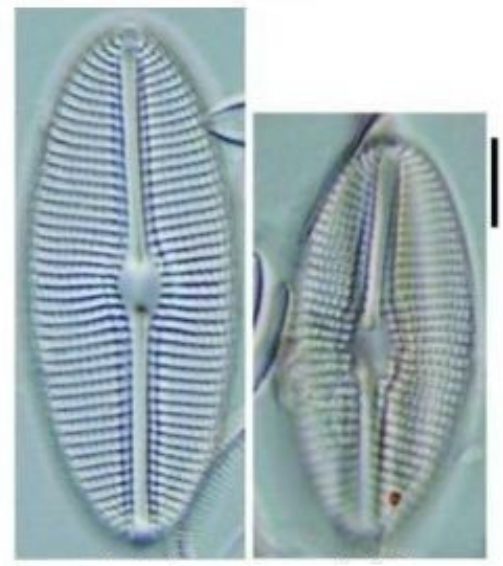

$\mathrm{n}$

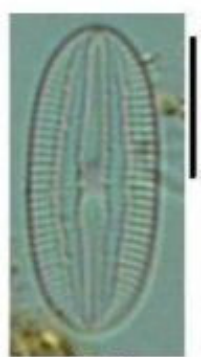

$\mathrm{O}$

Fig. 5. Epilithic diatoms (Bacillariophyceae): a, Brachysira intermedia; b, Brachysira neoexilis; c, Brachysira neglectissima; d, Cavinula variostriata; $\mathbf{e}$, Humidophila contenta; $\mathbf{f}$, Humidophila laevissima; $\mathbf{g}$, Humidophila perpusilla; h, Frustulia amphipleuroides; i, Frustulia saxonica; j, Frustulia vulgaris; k, Neidium affine; l, Luticola goeppertiana; m, Diploneis ovalis; $\mathbf{n}$, Diploneis elliptica; $\mathbf{0}$, Diploneis oculata. [Scale bars $6 \mu \mathrm{m}$. 

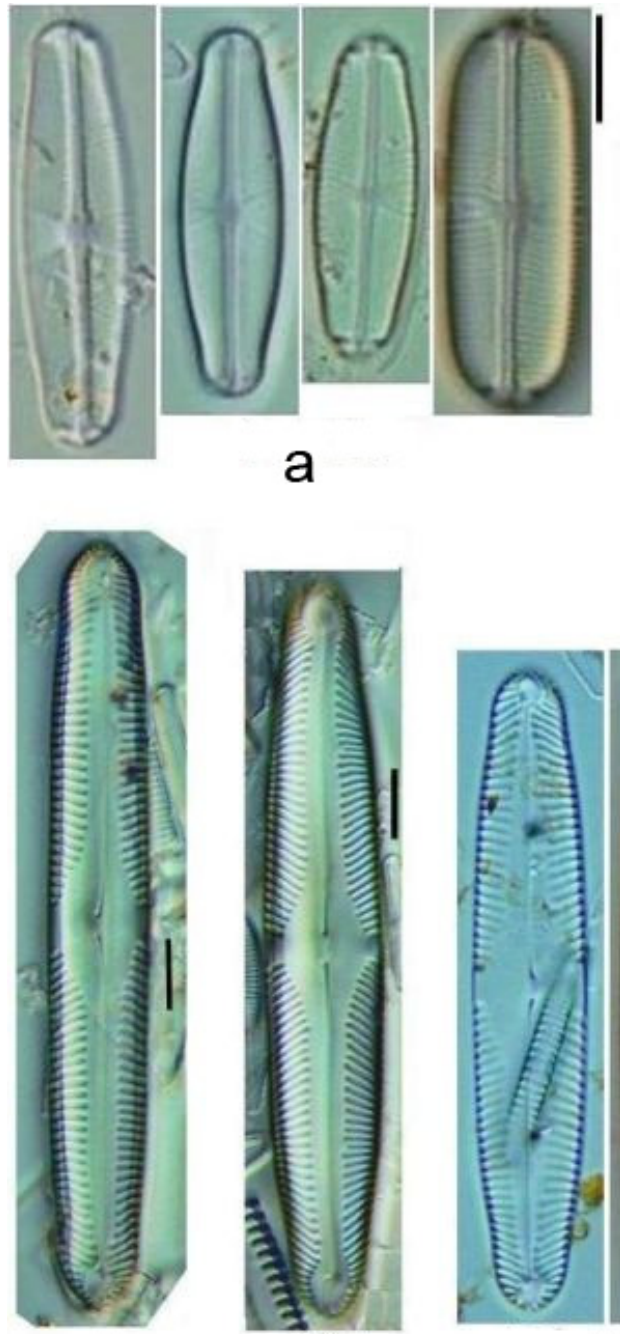

e
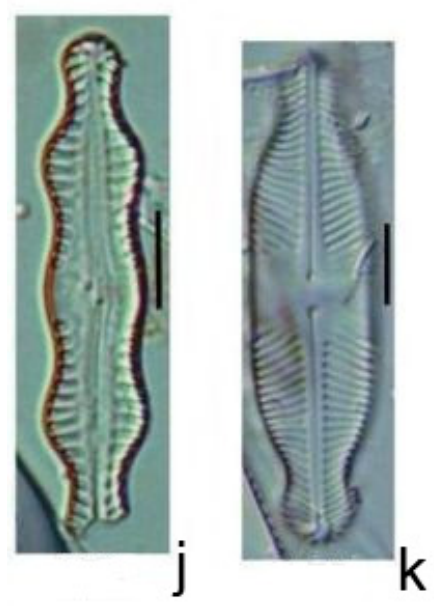

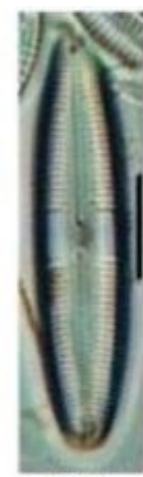

b

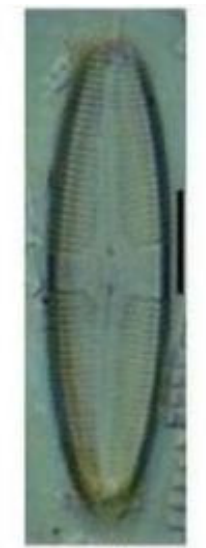

C

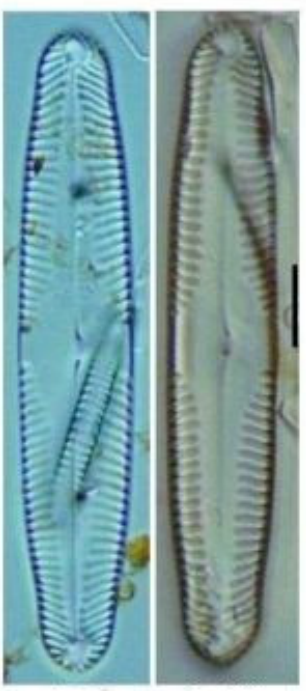

$f$

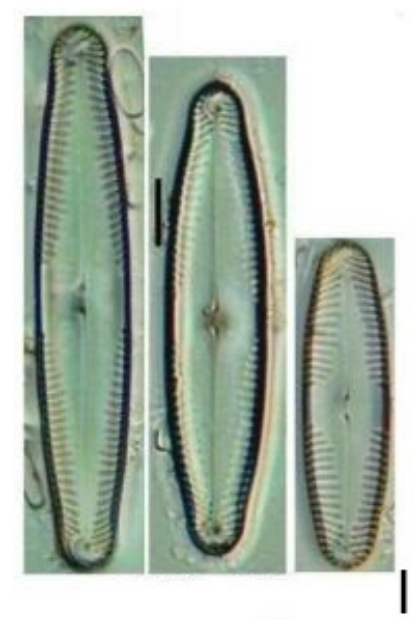

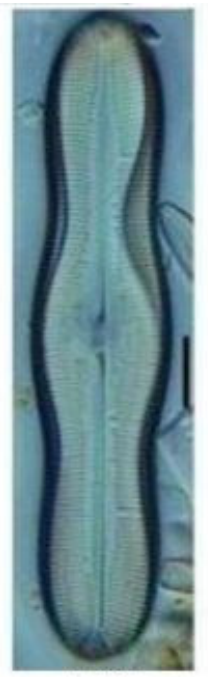

d
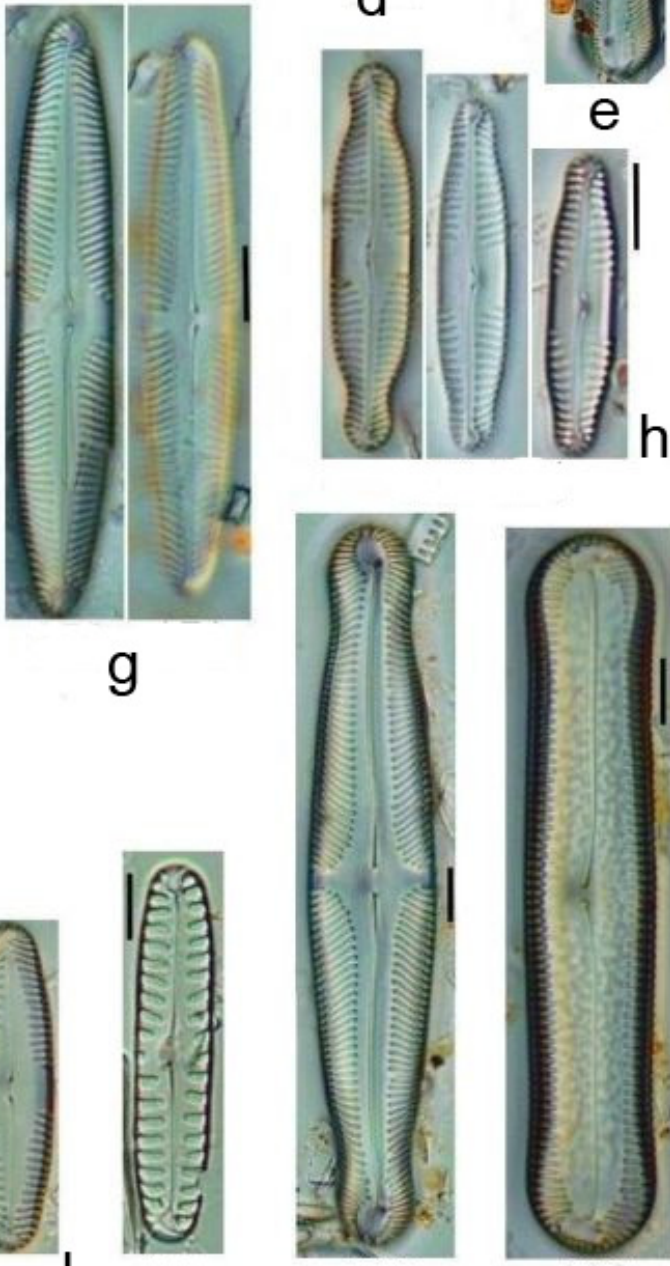

$\mathrm{n}$

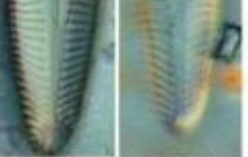

g

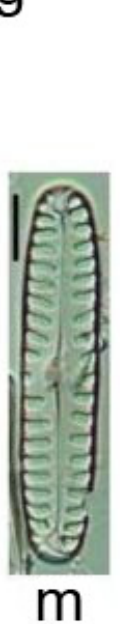

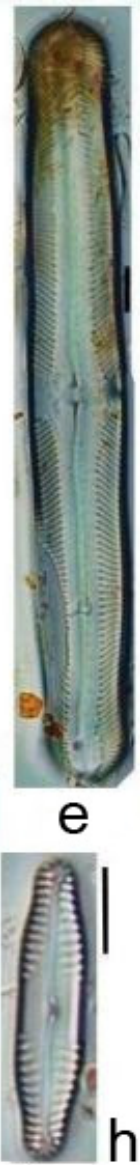

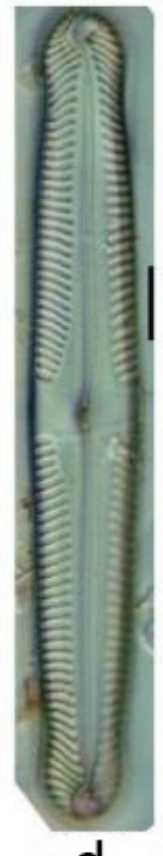

d
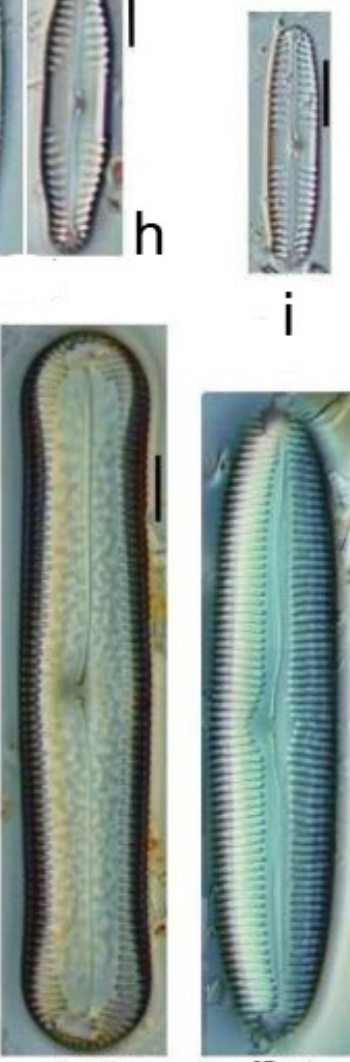

O

Fig. 6. Epilithic diatoms (Bacillariophyceae): a, Sellaphora pupula; $\mathbf{b}$, Caloneis bacillum; $\mathbf{c}$, Caloneis molaris; $\mathbf{d}$, Caloneis silicula; e, Pinnularia graciloides var. triundulata; d, Pinnularia subgibba var. undulata; e, Pinnularia divergens; f, Pinnularia parvulissima; $\mathbf{g}$, Pinnularia permicrostauron; $\mathbf{h}$, Pinnularia subcapitata; $\mathbf{i}$, Pinnularia sinistra; $\mathbf{j}$, Pinnularia nodosa var. angusta; k, Pinnularia lundii; $\mathbf{l}$, Pinnularia frauenbergiana; $\mathbf{m}$, Pinnularia borealis var. sublinearis; $\mathbf{n}$, Pinnularia divergens; o, Pinnularia acrosphaeria; p, Pinnularia stidolphii. [Scale bars $6 \mu \mathrm{m}$. 


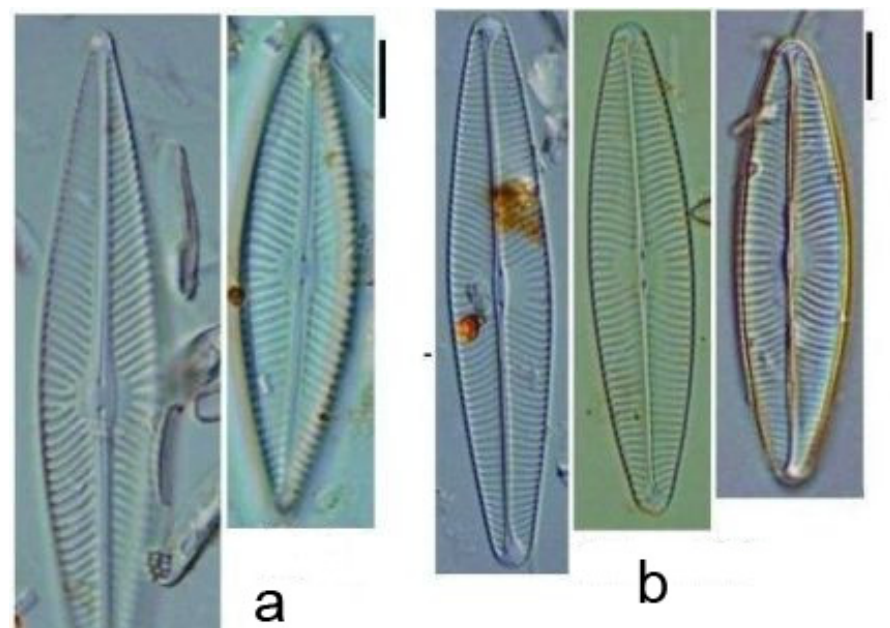

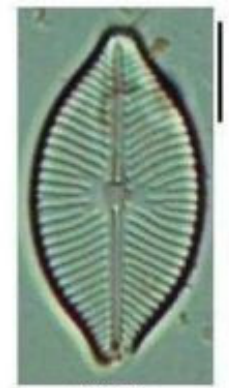

C

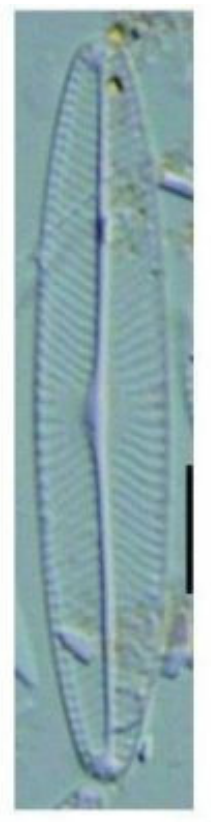

d

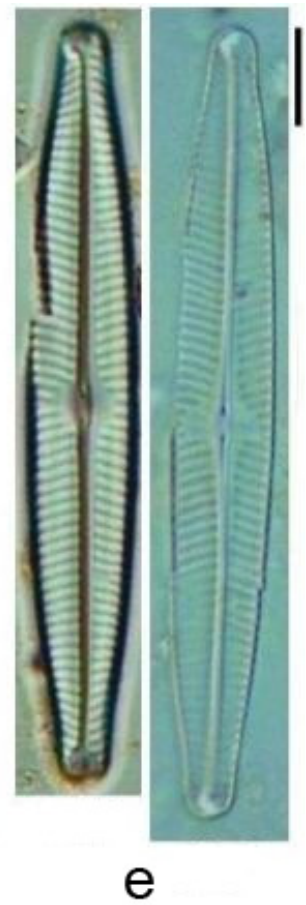

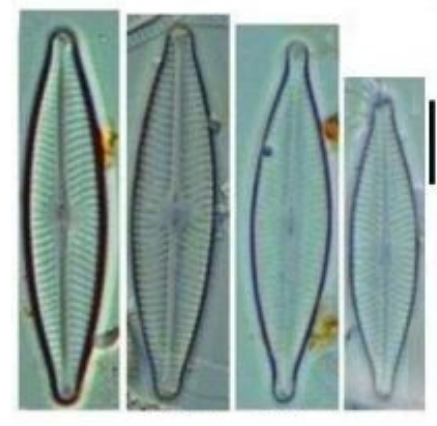

$\mathrm{f}$
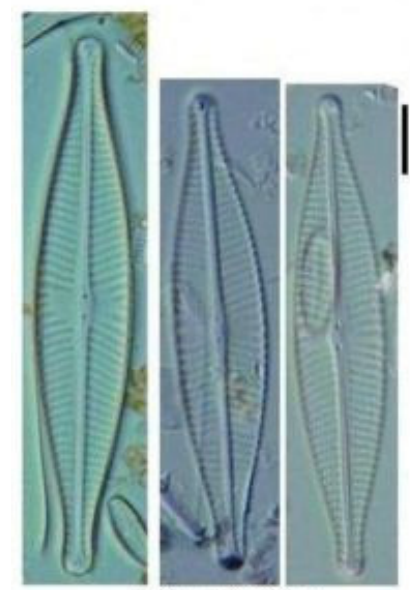

j

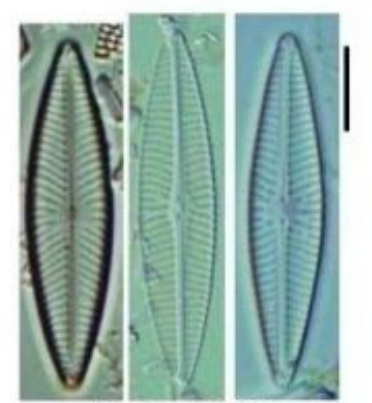

g
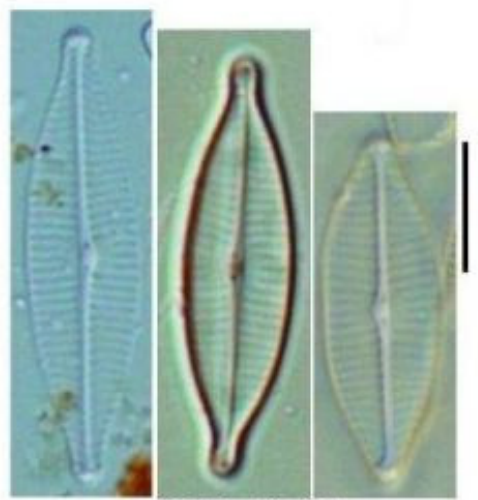

k

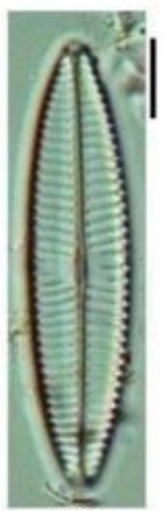

I

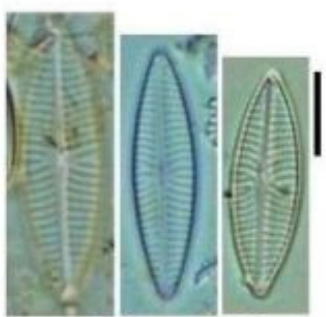

h

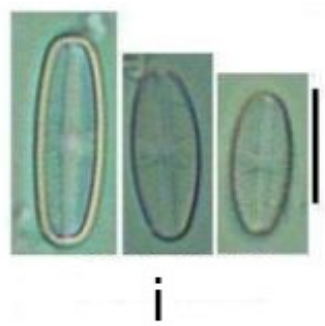

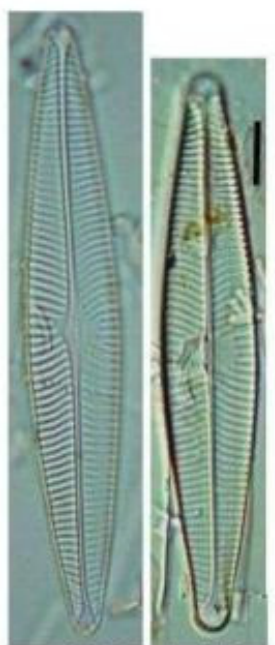

m

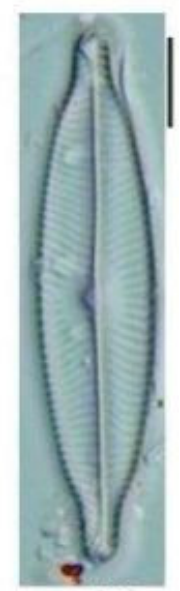

$\mathrm{n}$

Fig. 7. Epilithic diatoms (Bacillariophyceae): a, Navicula concentrica; b, Navicula lanceolata; $\mathbf{c}$, Navicula clementis; d, Navicula viridula; e, Navicula angusta; f, Navicula cryptocephala; g, Navicula cryptotenella; h, Navicula cryptotenelloides; i, Navicula minima; j, Navicula rhyncocephala; k, Navicula gregaria; $\mathbf{1}$, Navicula tripunctata; m, Navicula rostellata; $\mathbf{n}$, Navicula viridula. [Scale bars $6 \mu \mathrm{m}$.] 


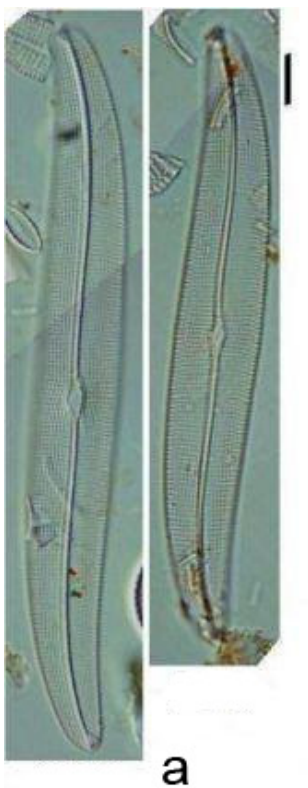

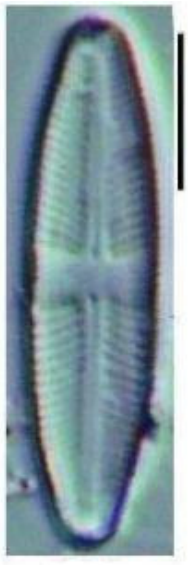

b
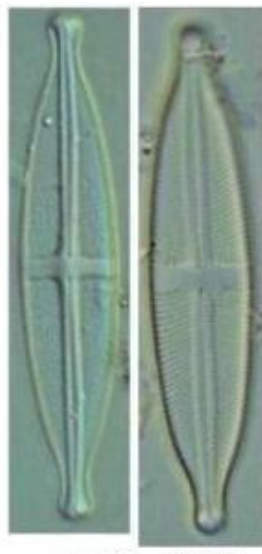

C
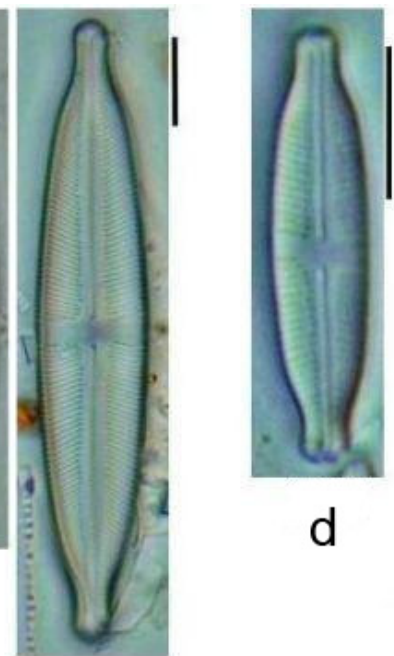

d

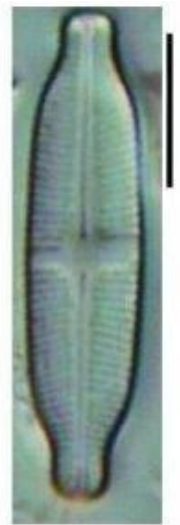

e

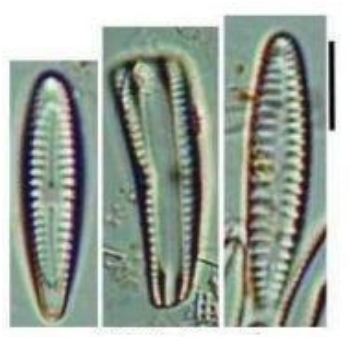

f

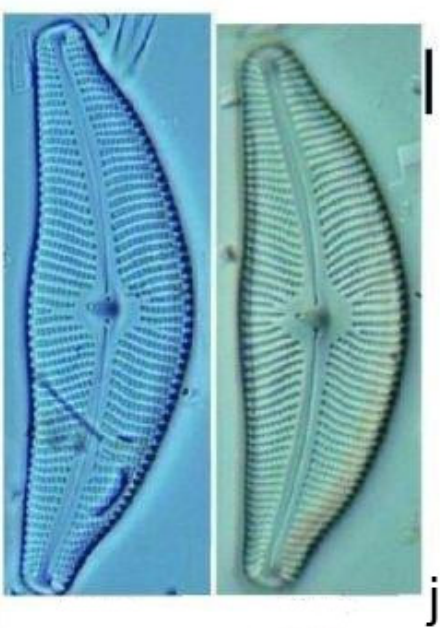

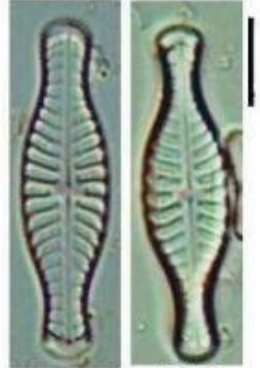

g

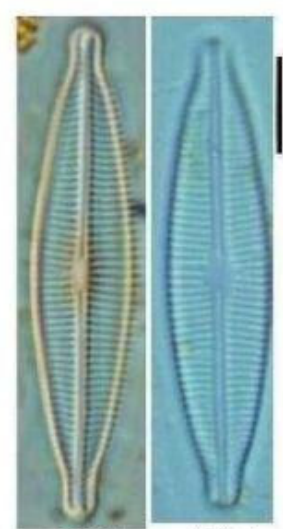

$\mathrm{h}$
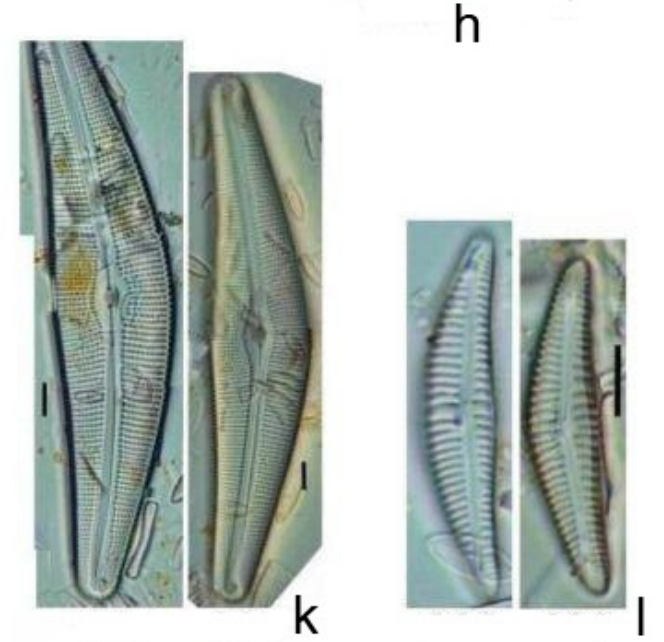
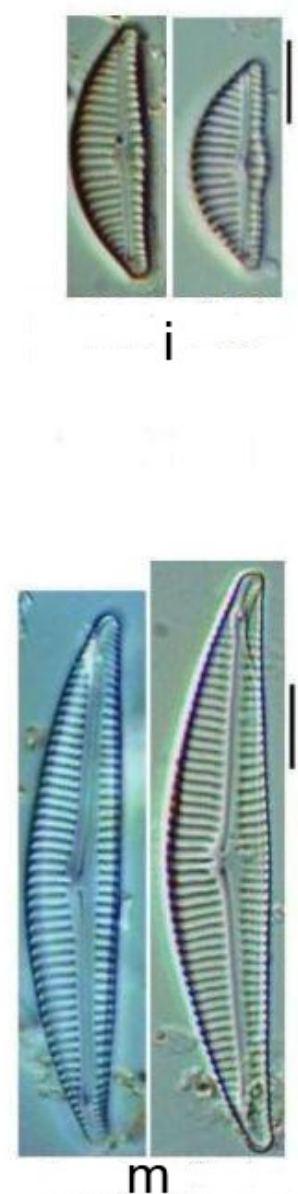

Fig. 8. Epilithic diatoms (Bacillariophyceae): a, Gyrosigma acuminatum; b, Stauroneis prominula; c, Stauroneis anceps; d, Stauroneis kriegerii; e, Stauroneis obtusa; f, Rhoicosphenia abbreviata; g, Hippodonta capitata; h, Craticula buderi; i,

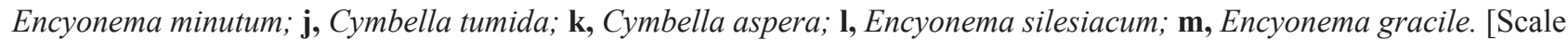
bars $6 \mu \mathrm{m}$. 


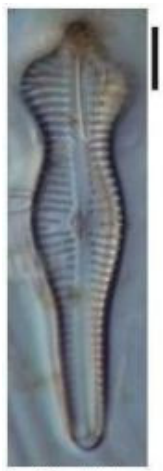

a

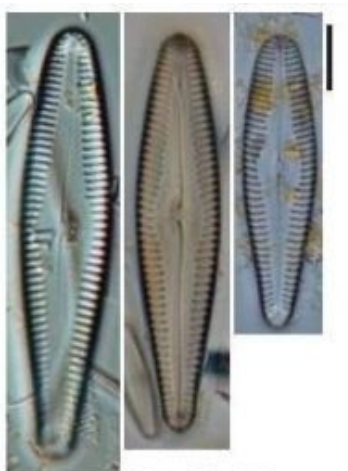

b

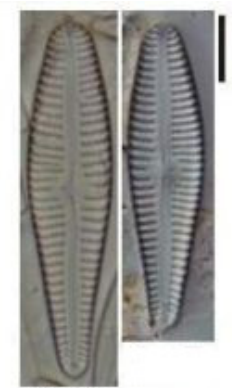

C

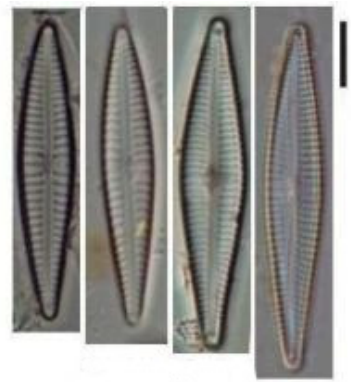

d

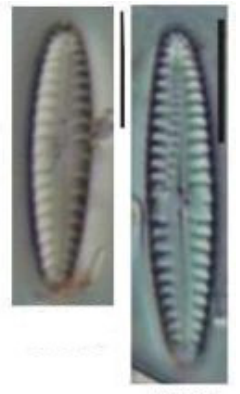

e

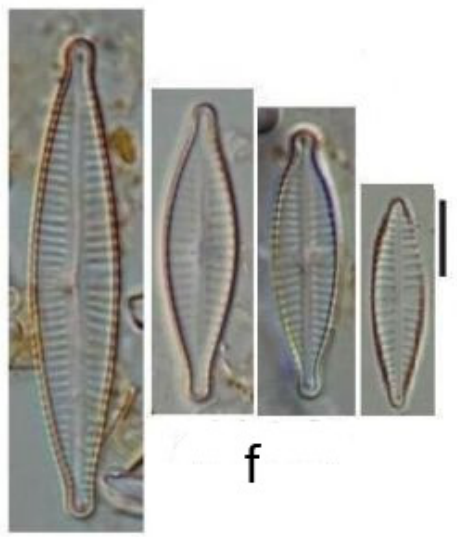

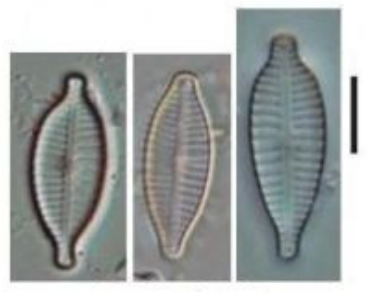

g

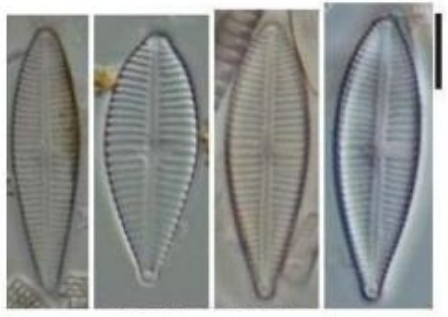

h
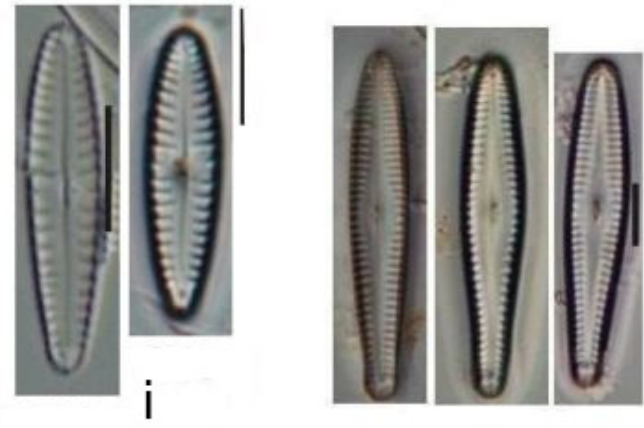

j

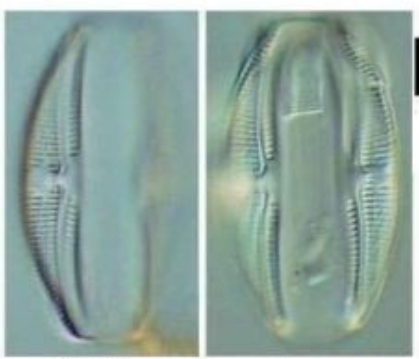

m

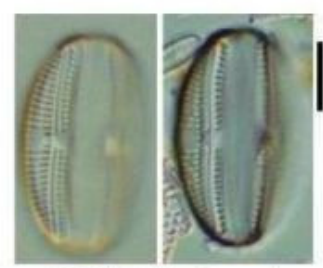

$\mathrm{n}$
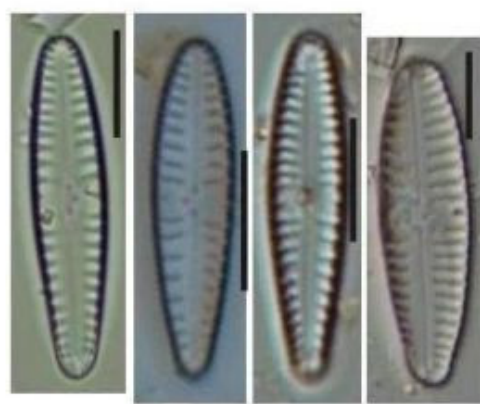

k

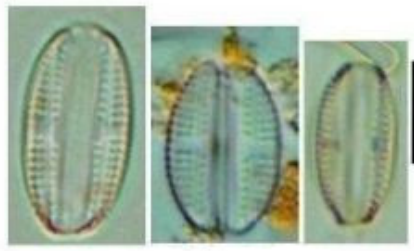

O
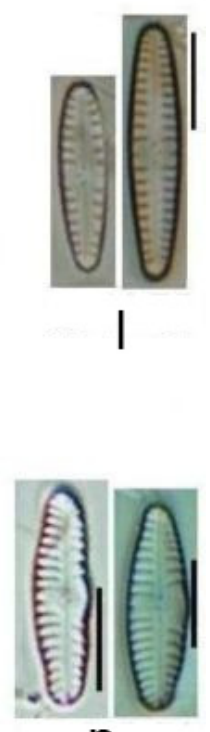

$p$

Fig. 9. Epilithic diatoms (Bacillariophyceae): a, Gomphonema acuminatum; b, Gomphonema ibericum; c, Gomphonema clavatum; d, Gomphonema gracile; e, Gomphonema minutum; f, Gomphonema parvulum var. exilissimum; g, Gomphonema parvulum var. parvulum; h, Gomphonema pseudoagur; i, Gomphonema pumilum; j, Gomphonema rhombicum; k, Gomphonema angustum; l, Gomphonema cf. pumilum; m, Amphora ovalis; n, Amphora inaeriensis; $\mathbf{0}$, Amphora pediculus; p, Reimeria sinuata. [Scale bars $6 \mu \mathrm{m}$. 

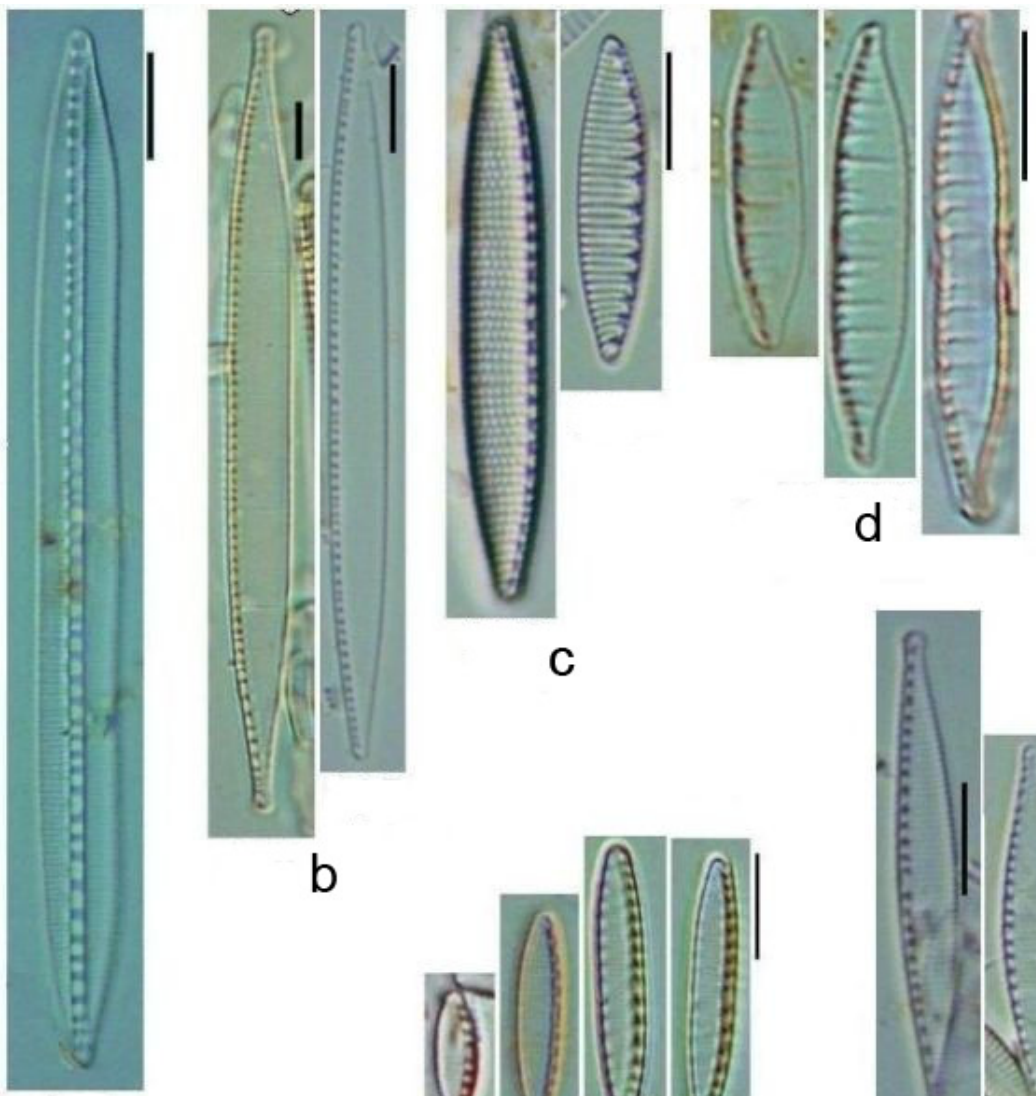

d

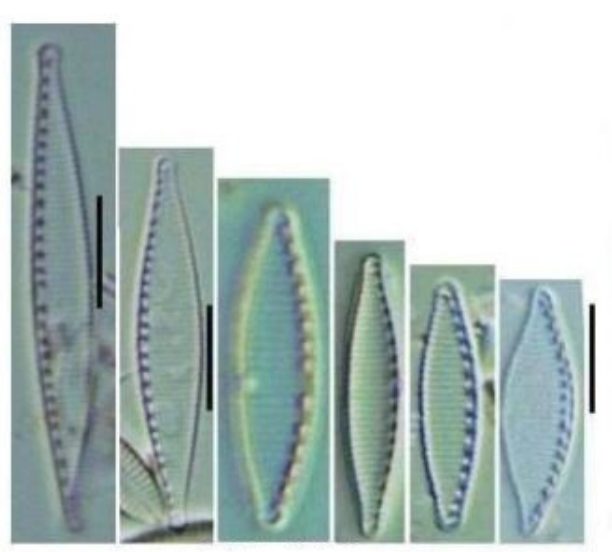

$\mathrm{h}$
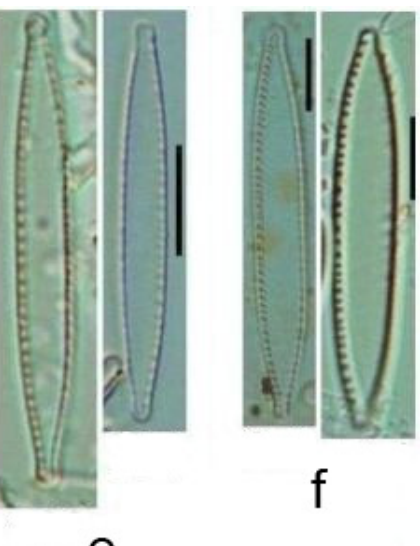

C

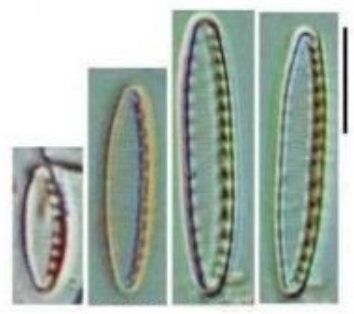

g
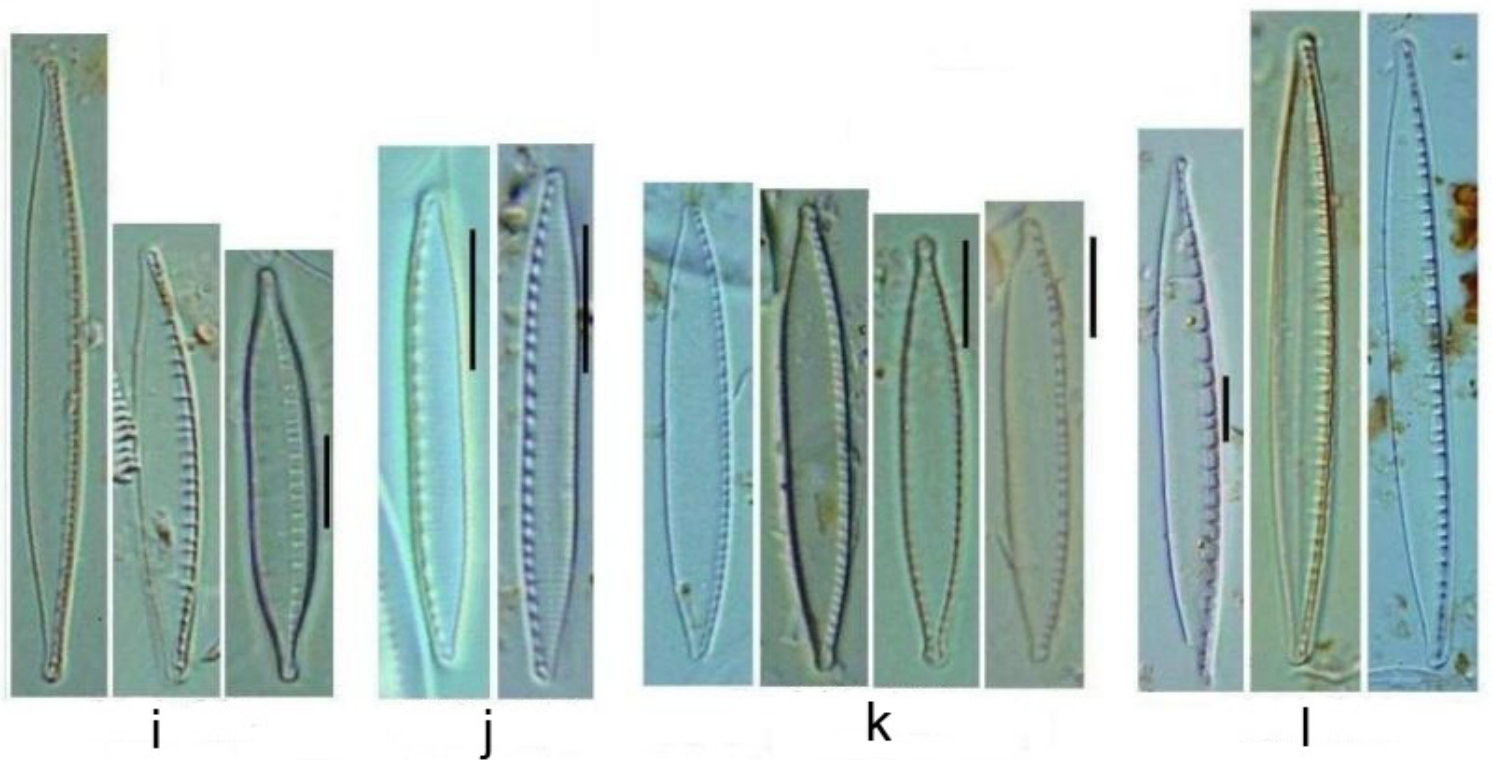

Fig. 10. Epilithic diatoms (Bacillariophyceae): a, Bacillaria paradoxa; b, Nitzschia sp.; c, Nitzschia amphibia; d, Nitzschia epithemoides; e, Nitzschia archibaldi; $\mathbf{f}$, Nitzschia palea var. debilis; $\mathbf{g}$, Nitzschia inconspicua; $\mathbf{h}$, Nitzschia

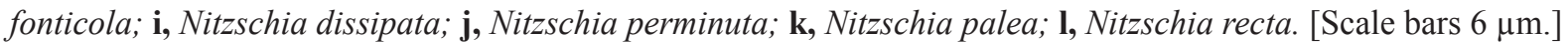


Eunotia subarcuatoides Alles, Norpel \& Lange-Bert. (fig. 3n)

Frustulia amphipleuroides (Grunow) Cleve (fig. 5h)

Frustulia saxonica Rabenh. (fig. 5i)

Frustulia vulgaris (Thwaites) De Toni (fig. 5j)

Gomphonema acuminatum Ehrenb. (fig. 9a)

Gomphonema angustum C.Agardh (fig. 9k)

Gomphonema gracile Ehrenb. (fig. 9d)

Gomphonema ibericum E.Reichardt (fig. 9b)

Gomphonema minutum (C.Agardh) C.Agardh (fig. 9e)

Gomphonema parvulum var. parvulum (Kütz.) Kütz. (fig. 9g)

Gomphonema parvulum var.exilissimum Grunow (fig. 9f)

Gomphonema pseudoaugur Lange-Bert. (fig. 9h) **

Gomphonema pumilum (Grunow) Reichardt \& Lange-Bert. (fig. 9i)*

Gomphonema rhombicum M.Schmidt (fig. 9j)

Grunowia solgensis (Cleve-Euler) Aboal (fig. 11e)

Gyrosigma acuminatum (Kutz.) Rabenh. (fig. 8a)*

Hantzschia amphioxys (Ehrenb.) Grunow (fig. 11c)

Hippodonta capitata (Ehrenb.) Lange-Bert., Metzeltin \& Witkowski (fig. 8g)

Humidophila contenta (Grunow) Lowe, Kociolek, J.R.Johansen, Van de Vijver, Lange-Bert. \& Kopalová (fig. 5e)

Humidophila perpusilla (Grunow) Lowe, Kociolek, Johansen, Van de Vijver, Lange-Bert. \& Kopalová (fig. 5g)

Humidophila laevissima (Cleve) Lowe, Kociolek, Johansen, Van de Vijver, Lange-Bert. \& Kopalová (fig. 5f)*

Iconella biseriata (Bréb.) Ruck \& Nakov (fig. 111)

Iconella helvetica (Brun) Ruck \& Nakov (fig. 11k)

Iconella linearis (W.Sm.) Ruck \& Nakov (fig. 11j)

Karayevia oblongella (Østrup) Aboal (fig. 4a)

Kolbesia suchlandtii (Hust.) Kingston (fig. 4b)

Lemnicola hungarica (Grunow) Round \& Basson (fig. 4c)

Luticola goeppertiana (Bleisch) D.G.Mann (fig. 51)

Navicula angusta Grunow (fig. 7e)

Navicula clementis Grunow (fig. 7c)*

Navicula concentrica Carter (fig. 7a)

Navicula cryptocephala Kütz. (fig. 7f)

Navicula cryptotenella Lange-Bert. (fig. $7 \mathrm{~g}$ )

Navicula cryptotenelloides Lange-Bert. (fig. 7h)

Navicula gregaria Donkin (fig. 7k)

Navicula lanceolata (C.Agardh) Ehrenb. (fig. 7b)

Navicula minima Grunow (fig. 7i)

Navicula rhyncocephala Kütz. (fig. 7j)

Navicula rostellata Kütz. (fig. $7 \mathrm{~m}$ )

Navicula tripunctata (O.F.Müll.) Bory (fig. 71)

Naviculadicta langebertalotii Cantonati \& Leira (fig. 12)***
Neidium affine (Ehrenb.) Pfitzer (fig. 5k)

Nitzschia amphibia Grunow (fig. 10c)

Nitzschia archibaldi Lange-Bert. (fig. 10e)

Nitzschia dissipata (Kütz.) Grunow (fig. 10i)

Nitzschia fonticola Grunow (fig. 10h)

Nitzschia inconspicua Grunow (fig. 10g)

Nitzschia nana Grunow (fig. 11b)

Nitzschia palea (Kütz.) W.Sm. (fig. 10k)

Nitzschia palea var. debilis (Kütz.) Grunow (fig. 10f)

Nitzschia recta Hantzsch (fig. 101)

Nitzschia sp. (fig. 10b)

Nupela lapidosa (Krasske) Lange-Bert. (fig. 4d)

Peronia erinacea Bréb. \& Arn. (fig. 3o)

Pinnularia parvulissima Krammer (fig. 6f)

Pinnularia acrosphaeria W.Sm. (fig. 6o)

Pinnularia borealis var. sublinearis Krammer (fig. 6m)

Pinnularia divergens W.Sm. (fig. 6e)***

Pinnularia lundii Hust. (fig. 6k)

Pinnularia sinistra Krammer (fig. 6i)***

Pinnularia stidolphii Krammer (fig. 6p)**

Pinnularia subcapitata W.Greg. (fig. 6h)

Planothidium daui (Foged) Lange-Bert. (fig. 4e)

Planothidium lanceolatum (Bréb. ex Kütz.) Lange Bert. (fig. 4f)

Planothidium peragalli (Brun \& Héribaud) Round \& Bukht. (fig. $4 \mathrm{~g})^{*}$

Psammothidium helveticum (Hust.) Bukht. \& Round (fig. 4h)

Psammothidium subatomoides (Hust.) Bukht. \& Round (fig. 4i)

Reimeria sinuata (W.Greg.) Kociolek \& Stoermer (fig. 9p)

Rhoicosphenia abbreviata (C.Agardh) Lange-Bert. (fig. 8f)

Sellaphora pupula (Kütz.) Mereschk. (fig. 6a)

Stauroneis anceps Ehrenb. (fig. 8c)

Stauroneis kriegerii Ehrenb. (fig. 8d)

Stauroneis obtusa Lagerst. (fig. 8e)

Stauroneis prominula (Grunow) Hust. (fig. 8b)*

Iconella delicatissima (F.W.Lewis) Ruck \& Nakov (fig. 11f)

Surirella angusta Kütz (fig. 11g)

Surirella brebissonii Krammer \& Lange-Bert. (fig. 11o)

Surirella patella Kutz. (fig. 11m)

Surirella roba L.Leclercq (fig. 11h)

Surirella robusta Ehrenb. (fig. 11i) 

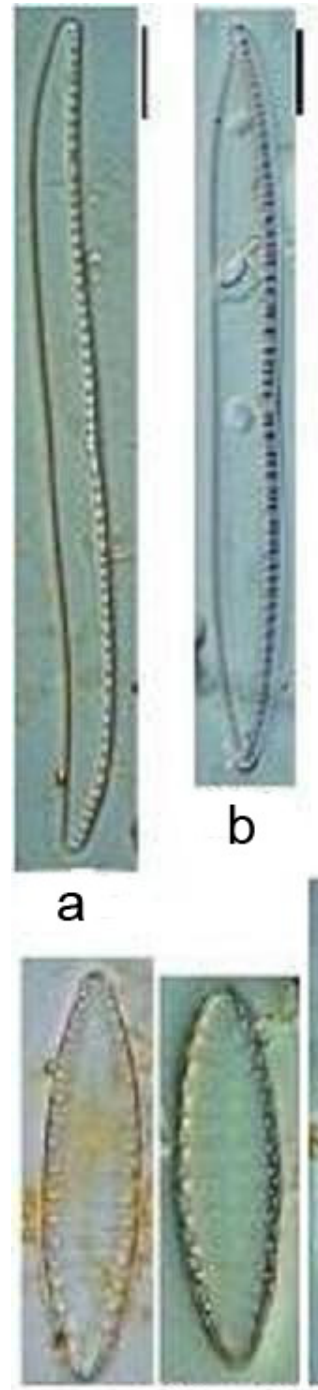

g

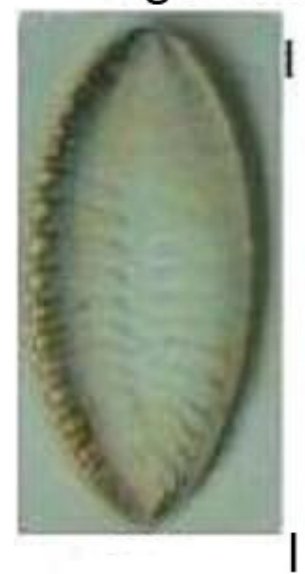

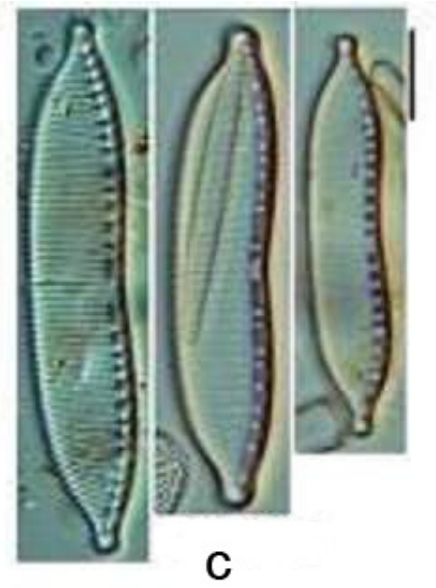
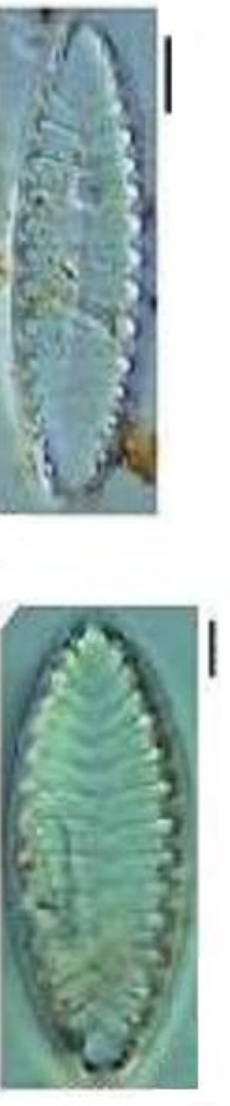

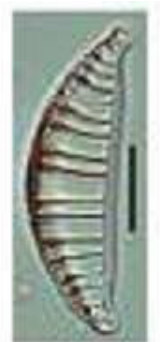

d

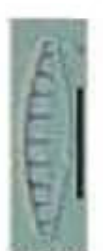

e
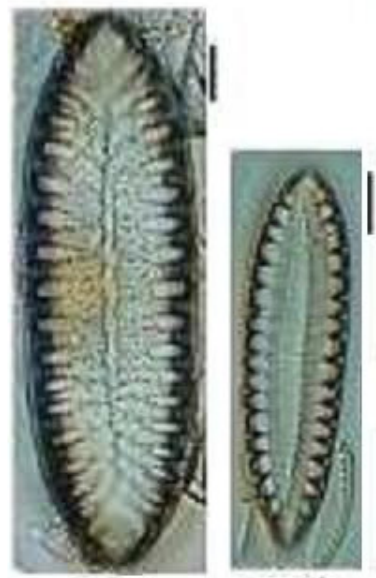

$\mathrm{h}$

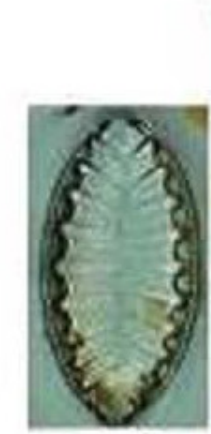

$\mathrm{m}$
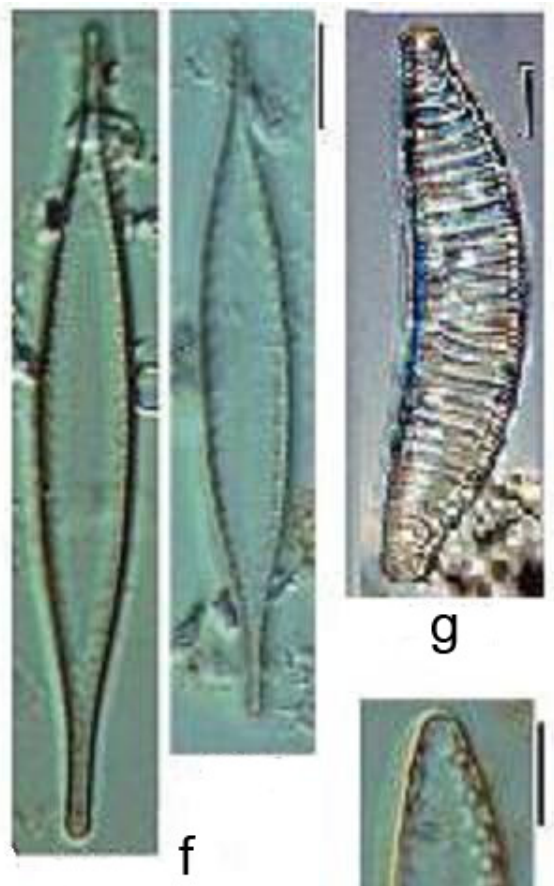

g
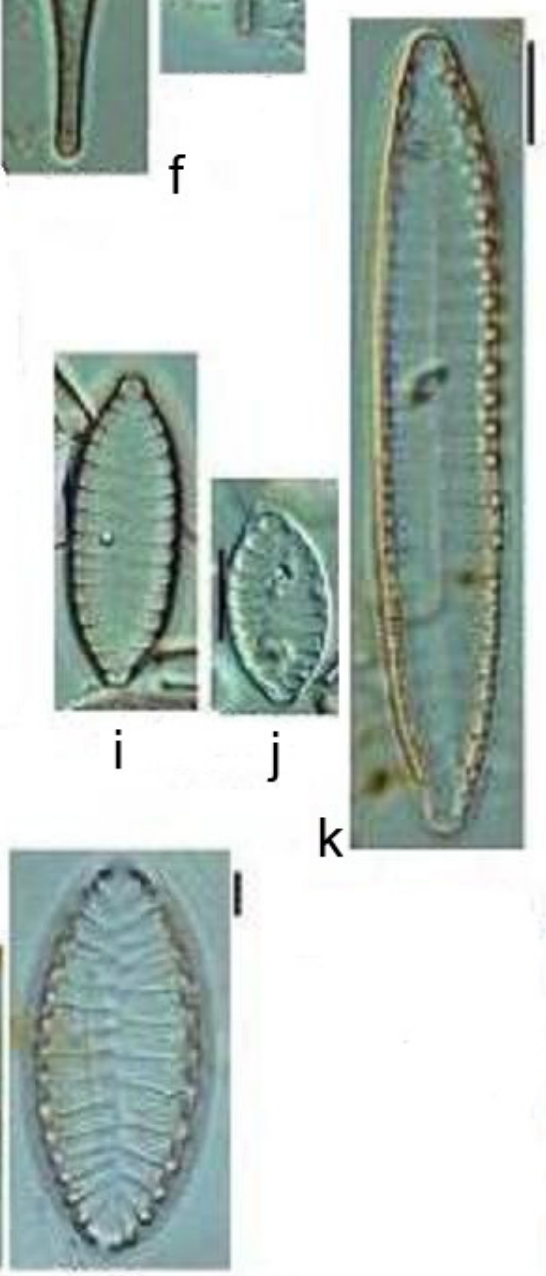

$\mathrm{O}$

Fig. 11. Epilithic diatoms (Bacillariophyceae): a, Nitzschia sigmoide; b, Nitzschia nana; c, Hantzschia amphioxys; d, Rhopalodia gibba; e, Grunowia solgensis; f, Iconella delicatissima; $\mathbf{g}$, Surirella angusta; $\mathbf{h}$, Surirella roba; i, Surirella robusta; j, Iconella linearis; k, Surirella helvetica; $\mathbf{1}$, Surirella biseriata; $\mathbf{m}$, Surirella patella; o, Surirella brebissonii. [Scale bars $6 \mu \mathrm{m}$.] 


\section{Description of selected taxa}

1. Achnanthes brevipes var. intermedia (Kütz.) Cleve, Kongl. Svenska Vetensk.-Akad. Handl. ser. 4, 27(3): 193 (1895). Fig. 4o.

Achnanthes intermedia Kütz., Alg. Aq. Dulc. Germ. 3: 3 (1833).

Achnanthes subsessilis Kütz., Alg. Aq. Dulc. Germ. 5: 3 (1833).

Valves $14-130 \times 9.5-40 \mu \mathrm{m}$, linear-lanceolate or linearelliptical to elliptical, with wedge-shaped or obtuse to broadly rounded endings, often a bit concave in the middle. Raphid valves with a moderately strong to weak curved raphe and equilateral-turning terminal branches. Axial area narrow, linear, while central area is fairly narrow, reaching to the edges forming a transverse fascia. Rapheless valves with narrow axial area, often running curved, mostly. Ends not wedge-shaped, but rather broadly rounded. Stria mostly 9-10 in $10 \mu \mathrm{m}$ and less coarse than Achnanthes brevipes C.Agardh.

Habitat and distribution.-This species has a cosmopolitan distribution along lowland areas even in estuaries. Hustedt (1930) thought that it could be regarded as caused by reduced salinity variation.
Note.-A similar taxon is Achnanthes taylorensis D.E.Kellogg, Stuiver, T.B.Kellogg \& G.H.Denton which has been often confused with Achnanthes brevipes var. intermedia. However, both differ in striae density (13-15 vs. 10 in $10 \mu \mathrm{m}$; Cleve 1895).

2. Brachysira intermedia (Østrup) Lange-Bert., Biblioth. Diatomol. 29: 34 (1994); Anomoeoneis intermedia Østrup, Danske Diat. 70, pl. II fig. 48 (1910). Fig. 5a.

Valves $25-33 \times 5-6.5 \mu \mathrm{m}$, outline very variable, strictly lanceolate, although gradually narrowing early to almost pointed ends. Striae $26-30$ in $10 \mu \mathrm{m}$, mostly from two to three composed lineolae, radial, and only at the ends sometimes parallel to slightly convergent. The Voigt unconformity is clearly visible on one side by truncated stria. Central area is small and indistinct rhombic, and clearly separated from the narrow linear axial area. Raphe filiform with small, clearly marked central pores.

Habitat and distribution.-Characteristic habitats are oligotrophic to dystrophic waters with very low electrolyte content on silicate soils, particularly in mountainous areas.

Note.-A similar taxon is Brachysira brebissonii R.Ross, but differs because of the duller, broader and not early narrowing ends. The
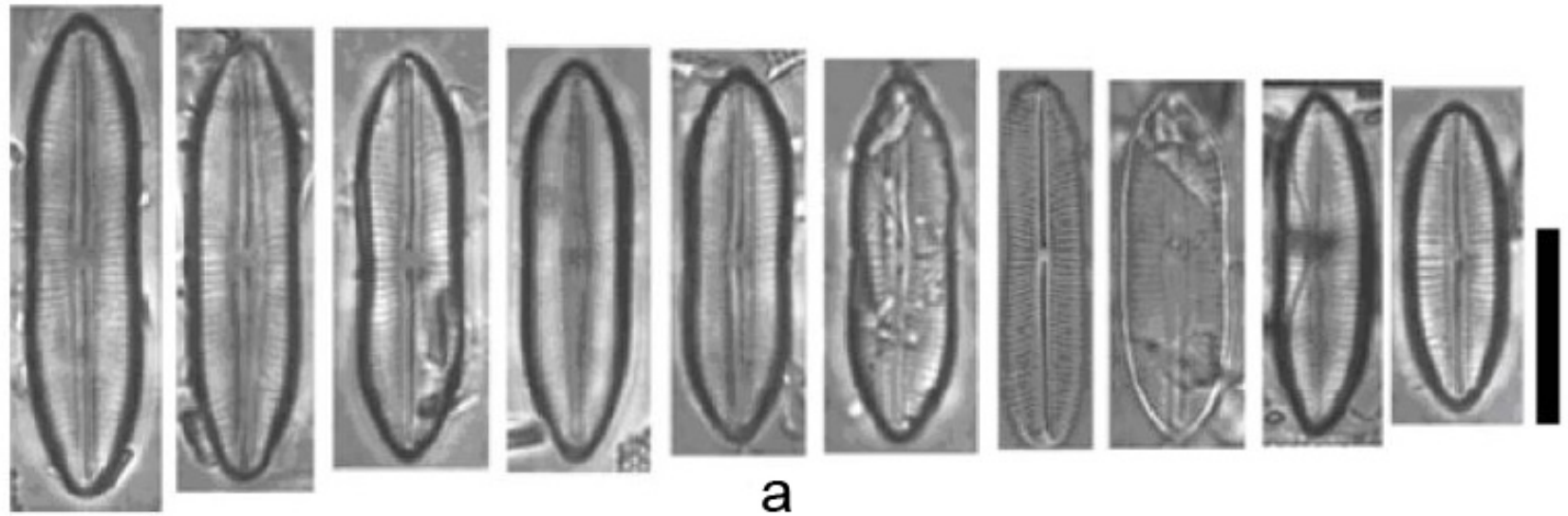

a
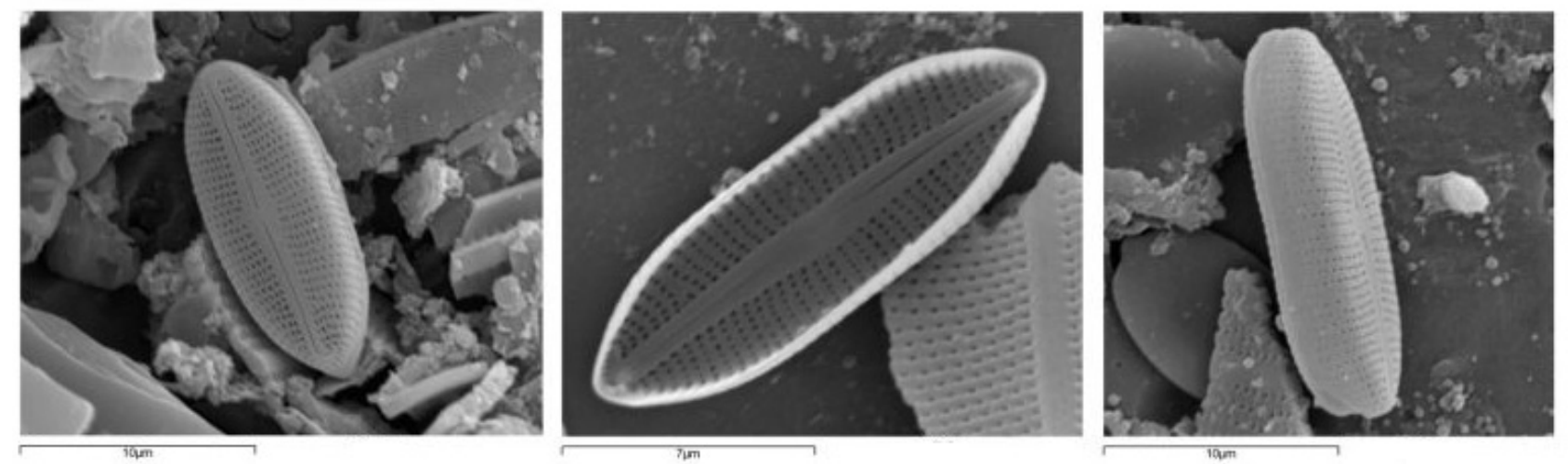

b

Fig. 12. Naviculadicta langebertalotii: a, light microscope images (scale bar $10 \mu \mathrm{m}$ ); b, SEM images (adapted from Cantonati \& al. 2012). 
distribution of this taxon in Europe is not known precisely because of the confussion with Brachysira brebissonii.

3. Cymbella aspera (Ehrenb.) Cleve, Kongl. Svenska Vetensk. Acad. Handl. ser. 4, 26 (2): 175 (1894); Cocconema asperum Ehrenb., Ber. Bekanntm. Verh. Königl. Preuss. Akad. Wiss. Berlin: 206 (1840); Cymbella lanceolata var. aspera (Ehrenb.) Brun., Diatom. Alpes 57, pl. 9, fig. 16 (1880); Cymbella aspera (Ehrenb.) H.Perag., J. Microgr.: 237 (1889); Cymbella aspera (Ehrenb.) Cleve, Öfvers. Kongl. Vetensk.-Akad. Förh. 26 (2): 175 (1894), comb. superfl. Fig. 8k.

Frustulia gastroides Kütz., Linnaea 8(5): 543, pl.13 fig.9 (1833); Cymbella gastroides (Kütz.) Bréb. \& Godey, Mém. Soc. Acad. Sci. Falaise.: 49 (1835); Cymbella gastroides (Kütz.) Kütz., Kieselschal. Bacill.: 79, pl. 6 fig. 4B (1844), comb. superfl

Valves $110-200 \times 26-35 \mu \mathrm{m}$, moderately to distinctly dorsiventral, dorsal margin evenly arched. Ventral margin slightly protuberant at the central part. Valve ends not protracted and broadly rounded. Axial area moderately wide, linear, widening at mid-valve to form a widened central area. Raphe filiform near the proximal ends with moderately large roundish central pores which are moderately distant and slightly ventrally deflected. Distal ends slightly reverse-lateral, terminal fissures sickleshaped. Striae slightly radiate. Puncta distinctly visible and more or less roundish. Striae $6.5-8$ in $10 \mu \mathrm{m}$.

Habitat and distribution.-Although widely distributed in Europe, it is not often recorded in large numbers. It prefers oligotrophic waters, especially favoured are habitats in the mountains of average electrolyte content.

Note.-It is distinguished from other similar taxa by outline, size, pearl-like puncta, and especially by the structure of the proximal raphe ends. Cymbella aspera has large roundish central pores, whereas Cymbella lanceolata (C.Agardh) C.Agardh has crosier-shaped central fissures. The puncta of Cymbella neogena (Grunow) Krammer are less shining "aspera", and this taxon has more than 12 puncta/10 $\mu \mathrm{m}$, and the central pores are less distantly arranged. The valves of Cymbella peraspera Krammer are larger - broader than $38 \mu \mathrm{m}-$. Cymbella subaspera Krammer is smaller, the outline is stockier and smaller forms have straight to slightly concave ventral margins.

4. Eunotia faba Ehrenb., Ber. Bekanntm. Verh. Königl. Preuss. Akad. Wiss.: 45 (1837); Epithemia faba (Ehrenb.) Kütz., Kieselschal. Bacill.: 36 (1844).

Valves are asymmetric to the apical axis and variably asymmetric to the transapical axis. Dorsal margins are moderately arched. Ventral margins are weakly concave. The apices are broadly rounded, with a deep notch on the ventral side. Terminal raphe fissures are very short at the junction of the valve face and mantle. Striae are radiate and very finely punctate. Areolae are difficult to resolve in LM.

Habitat and distribution.-In Europe Eunotia faba was found in Ireland, Wales and Romania from soft, somewhat dystrophic ponds, lakes and swamps. Camburn \& Charles (2000) reported this taxon - as Eunotia vanheurckii R.M.Patrick - from low-alkalinity lakes in the Adirondack Mountains of New York. Eunotia faba has been recorded from in anthropogenically undisturbed oligotrophic, weakly acidic to alkaline habitats in mountainous areas (Lange-Bertalot \& al. 2011).

Note.-A similar taxon is Eunotia incisa that has acutely rounded apices and narrower valves - less than $5 \mu \mathrm{m}$ wide-. Eunotia rhomboidea Hustedt is also heteropolar, or asymmetric to the transverse axis, but Eunotia faba has distinctly wider valves.

\section{Eunotia implicata Nörpel, Alles \& Lange-Bert., Nova} Hedwigia 53: 206 (1991).

Eunotia impressa var. angusta Grunow in Van Heurck, Syn. Diatom. Belgique, Atlas, pl. 33 fig. 22, pl. 35 fig.1 (1881).

Valves $20-40 \times 3-6 \mu \mathrm{m}$. Ventral margin is weakly concave, while dorsal margin is distinctly convex. Frequently, this dorsal margin presents two shallow undulations. Valve somewhat narrower at the ends than at the centre. The ends of the valve are rounded, narrowing slightly but they do not set off from the main body of the valve. The terminal nodes are located near but not at the end of the valve. Striae in the middle around 14-16 in $10 \mu \mathrm{m}$, about 22 in the ends; striae straight and almost perpendicular to the ventral side in the center of the valve.

Habitat and distribution.-This taxon is fairly common in silicatedominated streams of the highlands and the North German lowlands, occurring even at high numbers, but rarely observed in stagnant water (Hoffman \& al. 2013). The habitats are largely anthropogenically undisturbed, low in electrolytes and oligotrophic to dystrophic. OrtizLerín \& Jaume Cambra (2007) reported this taxon from low to upland streams in Northern Spain -76-1,356 m a.s.1. - with $\mathrm{pH}=4.3-7.9$ and conductivity ranging from 4.17 to $720 \mu \mathrm{S} \cdot \mathrm{cm}^{-1}$. Eunotia implicata is an acidophilus taxon, mainly occurring at $\mathrm{pH}$ below 7 (Van Damm \& al. 1994). In this work Eunotia implicata has a $\mathrm{pH}$ optimum of 6.3 and was found in oligotrophic lowland streams.

Note.-Eunotia implicata can be mistaken with exceptionally thin specimens of Eunotia minor (Kütz.) Grunow, but are distinguished by a higher striae density $-9-16 / 10 \mu \mathrm{m}-$ in the middle section.

6. Eunotia incisa W.Greg., Quart. J. Microscop. Sci. 12: 96, pl. 4 fig. 4 (1854). Fig. 3.

Valves $15-50 \times 4-7 \mu \mathrm{m}$. Striae $13-17$ in $10 \mu \mathrm{m}$, more distant at the centre of the valve than at the ends. Ventral margin straight in smaller specimens but weakly concave in larger specimens. Dorsal margin is convex. Apices are acutely rounded, with a "nose-like" appearance. Raphe distal ends lie on the valve mantle and the terminal raphe nodules are well set in from the apices. Frustules are rectangular to quadratic in girdle view.

Habitat and distribution.- It is one of the most common Eunotia species in Europe, probably a cosmopolitan species. Common in various electrolyte-poor, oligotrophic to dystrophic waters. Abundant in streams of the low mountain ranges of the study area but rare in the lowlands. Common in acidic, freshwater environments with low levels of organic matter and nutrients, and weakly mineralized. Occasionally it can occur in high abundances. In the north of Spain this species was found in habitats with $\mathrm{pH} 4.7-6.8$, conductivity $4.17-194 \mu \mathrm{S} \cdot \mathrm{cm}^{-1}$, although reached its maximum abudance at $\mathrm{pH} 5.3-6$, conductivity $38-51 \mu \mathrm{S} \cdot \mathrm{cm}^{-1}$, and altitude 472-484 m a.s.1. (Ortiz-Lerín \& Cambra 2007). In our study pH optimum was 6.3 and ranged between 5.5 and 6.8 . 
Note.-Several forms and variations in length, width, and stria density are currently included in the broad concept of Eunotia incisa. Lange-Bertalot \& al. (2011) have further described new species from Europe, such as Eunotia incisadistans Lange-Bert. \& Sienkiewicz, which has broader valves with lower stria density. Eunotia incisa is also similar to Eunotia boreoalpina Lange-Bert. \& Nörpel-Schempp and Eunotia rhomboidea Hust. only in girdle view. Eunotia veneris (Kütz.) De Toni was often confused in the older literature and is not detected in Central Europe. Valves of Eunotia incisa, however, are symmetric in regard to apex width and position of the helictoglossae compared to the asymmetry in these features in Eunotia rhomboidea. In North America, more differentiable taxa occur. Eunotia incisa is similar to Eunotia canicula P.C.Furey, R.L.Lowe \& J.R.Johans. and the size range of the two species overlap. The apices of Eunotia incisa, however, are more nose-like than the apices of Eunotia canicula. Furthermore, the valves of Eunotia canicula are narrower and the helicotoglossae are closer to the apex - less inset - as compared to Eunotia incisa. Individuals such as those in fig. 61 that have broader and rounded apices, such as valves with morphology similar to Taf. 161 figs. 13-15 in Krammer \& Lange-Bertalot (1991) could be considered as a new species, although for the moment they should be considered only as another variation of valve morphology.

7. Eunotia intermedia (Krasske ex Hust.) Nörpel \& Lange-Bert., Biblioth. Diatomol. 27: 32 (1993); Eunotia pectinalis var. minor f. intermedia Krasske ex Hust., Kieselalg. Deutschl. 2 (Lief. 2): 298, fig. 763 1-o (1932); Eunotia faba var. intermedia (Krasske ex Hust.) A.Cleve, Kongl. Svenska Vetensk.-Akad. Handl. 4 (1): 112, fig. 449 i-k (1933); Eunotia vanheurckii var. intermedia (Krasske ex Hust.) Patrick, Not. Nat. Acad. Nat. Sci. Philadelphia 312: 14 (1958). Fig. 3j.

Valves $14-45 \times 3.5-5 \mu \mathrm{m}$, thickened midway between the center and the ends. Striae 14-19 in $10 \mu \mathrm{m}$, getting closer to the ends. The ventral side of the valve is almost straight or weakly concave at most. The dorsal margin is convex. The apices hardly differ from the body of the valve. The terminal nodes are clearly differentiated, and located near the ends.

Habitat and distribution.-Spread, so far known with certainty only from the northern hemisphere, scattered in the area, mostly occurring in poor populations. Ortíz-Lerín \& Cambra (2007) found this taxon in habitats with $\mathrm{pH} 5.4-7.3$, and low conductivity $-17.6-112 \mu \mathrm{S} \cdot \mathrm{cm}^{-1}$ - in mid-altitude sites - 205-1,086 m a.s.l- - Acidophilus, mainly occurring at $\mathrm{pH}$ below 7 (Van Damm \& al. 1994). In the studied area the species has a $\mathrm{pH}$ optimum of 6.2 .

Note.-Similar outline to Eunotia faba. However the location of the terminal nodes is different.

8. Eunotia pectinalis var. undulata (Ralfs) Rabenh., Fl. Eur. Alg. 1: 73 (1864); Fragilaria pectinalis var. undulata Ralfs, Ann. Mag. Nat. Hist. 12: 107, pl. 2, fig. 3d (1843); Himantidium undulatum (Ralfs) W.Sm., Syn. Brit. Diat. 2: 112, pl. 33, fig. 28 (1856); Himantidium pectinale var. undulatum (Ralfs) Grun., Verh. K. K. Zool.-Bot. Ges. Wien 27: 341 (1862); Eunotia pectinalis f. undulata (Ralfs) A.Berg, Bot. Not. 426 (1939). Fig. 3k.

Valves $10-140 \times 5-10 \mu \mathrm{m}$, dorsiventral and symmetric to the transapical axis. Striae 7-15 in $10 \mu \mathrm{m}$, extending across the entire surface of the valve. Ventral margin straight or slightly concave, can appear biarcuate due to inflated central region. The apices of the valves have a rounded end. Raphe runs mainly on the valve mantle and at the poles and it is curved slightly over the face of the valve at the apices. Terminal nodes are clearly distinctive. Rectangular frustules in girdle view.

Habitat and distribution.-This taxon is not very well represented in this region, it was found only in six sites with low abundance. Often in circumneutral to weakly acidic, low conductivity waters (Patrick \& Reimer 1966)

Note.-This variety of Eunotia pectinalis has many, slight undulations along the dorsal margin and a central swelling to the ventral margin. These characters are used to distinguish the variety Eunotia pectinalis var. undulata from its nominate variety (Patrick \& Reimer 1966; Krammer \& Lange-Bertalot 1991)

9. Eunotia praerupta Ehrenb., Abh. Königl. Akad. Wiss. Berlin 1841: 414 (1843); Eunotia bidens var. praerupta (Ehrenb.) Aysel, J. Black Sea Medit. Environm. 11: 91 (2005).

Eunotia praerupta var. curta Grunow, Syn. Diatom. Belgique, pl. 34 (1881); Eunotia praerupta f. curta (Grunow) A.Cleve, Bih. Kongl. Svenska Vetensk.-Akad. Handl. 21: 33 (1895); Eunotia praerupta f. curta (Grunow) Mayer, Denkschr. Königl.-Baier. Bot. Ges. Regensburg. I: pl. 2, fig. 11 (1917), comb. superfl.; Eunotia praerupta-monos var. curta (Grunow) Cleve-Euler, Acta Geogr. 10(1): 16 (1948); Eunotia praemonos var. curta (Grunow) Cleve-Euler, Kongl. Svenska Vetensk.-Akad. Handl. ser. IV, 4(1): 115, fig. 452v (1953). Fig. 31.

Valves $20-100 \times 4-15 \mu \mathrm{m}$. Striae at mid-valve $6-13$ in $10 \mu \mathrm{m}$, irregularly and distantly spaced, and slightly more dense at valve ends, parallel, becoming strongly radiate at valve apices. Valves weakly to strongly curved with clearly protracted, broadly rostrate or abruptly terminated ends. The dorsal margin is convex; narrowed, and the ends are truncate-rostrate. The terminal nodules are distinct, at the ends of the valve, extending upwards along the apices. The frustles in the girdle view are rectangular.

Habitat and distribution.-Usually in mountainous localities in acid to circumneutral waters (Patrick \& Reimer 1966). Acidophilus: mainly occurring at $\mathrm{pH}<7$ (Van Damm \& al. 1994).

Note.-This species can be easily recognized by the convexity of the dorsal margin and by the characteristic truncate-rostrate to slightly capitate ends.

10. Eunotia subarcuatoides Alles, Norpel \& Lange-Bert, Nova Hedwigia 53: 188, pl. 4 figs. 1-36 (1991). Fig. 3n.

Valves 6-35(40) × 2.7-4.5 $\mu \mathrm{m}$, curved dorsiventrally and symmetric to the transapical axis. Dorsal margin consistently strongly convex, smooth, rarely linear. Ventral valve margin consistently weakly concave. Valve slightly narrowed towards the end. Apices rounded to slightly (sub-) rostrate. Striae 18-23 in $10 \mu \mathrm{m}$, extending across the entire valve face. Raphe slightly developed mainly on the valve mantle and restricted to the poles. Terminal nodules small, dot-like, positioned slightly distant from both valve apices 
and ventral valve margin. Frustules box-like or rectangular in girdle view. Raphe often only visible in girdle view.

Habitat and distribution.-It is a common diatom in Galicia rivers occuring in high abundance. Its highest abundance was at pH 6.1. Eunotia subarcuatoides has been classified as an acidobiontic with an optima occurrence at $\mathrm{pH}<5.5$ (Van Damm \& al. 1994). Anyway, it seems to tolerate high and strong variations of $\mathrm{pH}$ values (Alles \& al. 1991; OrtizLerín \& Cambra 2007).

11. Frustulia saxonica Rabenh., Süssw.-Diat. 61: 50, pl. 7, fig. 1 (1853); Navicula saxonica (Rabenh) Lagerstedt, Bih. Kongl. Svenska Vetensk.-Akad. Handl. 1 (14): 32 (1873); Frustulia rhomboides var. saxonica (Rabenh.) De Toni, Syll. Alg. 2: 277 (1891); Vanheurckia rhomboides var. saxonica (Rabenh.) Holmboe, Arch. Math. Naturv. 22(1): 44 (1899); Navicula rhomboides var. saxonica (Rabenh.) Budde, Arch. Hydrobiol. 19: 510 (1928). Fig. 5 i.

Valves $28-105 \times 10-18 \mu \mathrm{m}$, generally rhomboid in shape, although valves at the small end of the size range are not strongly rhomboid. Striae $29-32 / 10 \mu \mathrm{m}$, parallel in the middle to gradually strongly convergent to the end, radiate at the apices; longitudinal striae are present, but may be disorganized at the valve center. The apices are slightly constricted and narrowly rounded. The longitudinal ribs are slightly curved. Both the thickness of the ribs and size of the central nodule are variable in relation to valve size. The porte-crayon is relatively small.

Habitat and distribution.-This is a characteristic species from dystrophic and low-electrolyte waters. Indicator of high ecological quality.

Note.-Frustulia crassinervia (Bréb.) Lange-Bert. \& Krammer has prominently undulate valve margins, while the margins of Frustulia inculta P.Siver, J.Pelczar \& P.Ham. are very slighty undulate. In addition, the valve margins of Frustulia crassinervia are more strongly undulate and its apices more narrowly protracted than those in Frustulia saxonica.

12. Humidophila perpusilla (Grunow) Lowe, Kociolek, Johansen, Van de Vijver, Lange-Bert. \& Kopalová, Diatom Res. 29 (4): 358 (2014); Navicula perpusilla Grunow, Verh. K. K. Zool.-Bot. Ges. Wien 10: 552, pl. 2 fig. 7 (1860); Schizonema perpusillum (Grunow) Kuntze, Revis. Gen. Pl. P. 3 (3): 554 (1898); Navicula gallica var. perpusilla (Grunow) Lange-Bert., Biblioth. Diatomol. 9: 71 (1985); Diadesmis perpusilla (Grunow) D.G.Mann, Diatoms: 666 (1990); Diadesmis gallica var. perpusilla (Grunow) LangeBert., Iconogr. Diatomol. 2: 45 (1996). Fig. 5g.

Navicula flotowii Grunow, Syn. Diatom. Belgique: 109, pl. 14 fig. 41 (1880); Navicula perpusilla var. flotowii (Grunow) J.B.Petersen, Bot. Iceland. 2(2): 328-447 (1928).

Valves 6-14 $\times 4-5 \mu \mathrm{m}$, elliptical in the smaller specimens and linear in the larger, and centrally inflated. Striae 24-30 in $10 \mu \mathrm{m}$. Flat valves with large sternum raphe, and two rows of elongated areolae that are only visible in LM. A longitudinal row of areolae runs over the mantle along the margin of the valve, interrupted at the ends.

Habitat and distribution.-Humidophila perpusilla is a widespread diatom common in silicate dominated waters in highlands. It has also been cited from aerial habitats and often occurring in association with Diadesmis contenta (Grunow) D.G.Mann. Characteristic are also habitats with reduced light intensity such as caves and rock crevices. The freshwater genus Humidophila Lowe \& al. is typically restricted to subaerial habitats (Round \& al. 1990). In our study, Humidophila perpusilla was found in poor electrolyte, circumneutral and oligotrophic waters.

Note.-The complex of species around Diadesmis contenta s.1. was reviewed in part by Moser \& al. (1998). Lowe \& al. (2014) proposed the genus Humidophila to accommodate a number of taxa formerly classified within the subgenus Paradiadesmis Lange-Bert. \& Le Cohu. Based on valve morphology analyses with scanning electron microscopy and microhabitat preferences, Diadesmis perpusilla was transferred to Humidophila. Our specimens most closely resemble Humidophila perpusilla as illustrated in Krammer \& Lange-Bertalot (1986) as Navicula gallica var. perpusilla.

\section{Naviculadicta langebertalotii Cantonati \& Leira, Nova} Hedwigia 141: 73, figs. 1-3 (2012).

Valves $12-26 \times 4.5-5.5 \mu \mathrm{m}$. Striae $16-23$ in $10 \mu \mathrm{m}$, parallel in the central part, soon becoming moderately radial, becoming parallel again towards the apices. Raphe branches straight, filiform; central endings only very slightly bent; proximal raphe ends relatively close to each other, and only very slightly bent in the same direction which is opposite to that one towards which the terminal fissures are curved. Faint longitudinal lines visible on both sides of the raphe. Axial area narrow and straight, slightly widening towards the central area that is just a small unilateral expansion of the axial area.

Habitat and distribution.-The type locality - Soutomaior, at the River Verdugo - is a low altitude - $140 \mathrm{~m}$ a.s.1. - stream stretch located in a narrow valley with steep slopes. The river bed is dominated by hard geological substrata, such as granites and phyllites. This species is typical of low-conductivity, meso-eutrophic, slightly-acidic but nonacidified, running-water sites, that reaches maximum relative abundances in autumn.

Note.-The most similar Navicula s.1. species are Navicula natchikae J.B.Petersen and Navicula oregonensis Hustedt. Both are clearly larger, and have parallel straight margins. Striae are interrupted close to the valve margin by a longitudinal rib developing parallel to the valve margin.

14. Pinnularia divergens W.Sm., Syn. Brit. Diat. 1: 57, pl. 18 fig. 177 (1853); Navicula divergens (W.Sm.) Grunow, Verh. K. Zool.-Bot. Ges. Wien 10: 523 (1860); Stauroptera divergens (W.Sm.) O.Kirchn., Alg. Schles.: 177 (1878); Schizonema schweinfurthii Kuntze, Rev. Gen. Pl. 3 (3): 550 (1898). Fig. 6e.

Valves 50-126 × 12-22 $\mu \mathrm{m}$, linear, lanceolate, linearelliptical, sides parallel, slightly convex or triundulate, ends broadly rounded or broadly rostrate to subcapitate. Raphe lateral, outer fissure somewhat curved, commonly filiform in the middle, central pores with lateral annexes, terminal fissures broadly bayonet-shaped. Axial area linear or linear-lanceolate and rhombic to rounded-rhombic 
central area extending to a small fascia that reaches the valve margin. Striae 9-14 in $10 \mu \mathrm{m}$, moderately to strongly radiate in the middle, moderately to strongly convergent at the ends, longitudinal bands absent, the alveoli completely open to the inside. Two conical spots which appears dark in the light microscope, each one at the edges of the central area which correspond to wall thickenings.

Habitat and distribution.-Pinnularia divergens was found at five study sites: the Ouro, Sor, Eume, Traba, and Sar rivers. All of the sites are oligotrophic waters from mountainous areas with a $\mathrm{pH}$ below 7 and low-electrolyte content.

Note.-At least eleven varieties exist in the literature, though some of these merely represent life-cycle stages. Striae divergence criterion, bayonet-shaped terminal fissures, and conical spots in the small fascia are important characters (Krammer 2000).

15. Pinnularia sinistra Krammer, Biblioth. Diatomol. 26: 175, pl. 37 fig. 1-16 (1992). Fig. 6i.

Valves $17-52 \times 4-6.5 \mu \mathrm{m}$. Striae (11)13(14) in $10 \mu \mathrm{m}$. The central area consists of a moderately broad, often asymmetric, fascia. The specimens have valve ends broadly protracted and subrostrate nearly as wide as the valve.

Habitat and distribution.--Specimens were found at the Furelos river. The Furelos river is a tributary of the Ulla on its right bank. The river is located in the southeast corner of the province of A Coruña - belonging to Terra de Melide - , almost in the center of Galicia, and it flows through ultramafic rocks - the largest outcrop in the Iberian northwest-. The site is located at medium altitude $-700 \mathrm{~m}$ a.s.1. — at $25 \mathrm{~km}$ from the source with an upstream drainage area of $150 \mathrm{~km}^{2}$. Pinnularia sinistra was found when the stream was at high flow condition. Pinnularia sinistra is cosmopolitan in the palaearctic region. Very common and locally abundant in oligotrophic electrolyte-poor, acidic streams. Tolerant to anthropogenic acidification (Hoffmann \& al. 2011).

16. Pinnularia stidolphii Krammer, Diatoms 1: 231, pl. 134 figs. 1-7, pl. 183 fig. 3 (2000). Fig. 6p.

Valves $82-133 \times 18-20 \mu \mathrm{m}$, linear, with sides almost parallel to slightly convex. Striae $7-9$ in $10 \mu \mathrm{m}$, radiate in the centre zone, becoming slightly convergent close to the poles, and crossed by a small longitudinal strip. Apex broadly rounded and slightly narrowed. Longitudinal area is linear and moderately wide. Central area is asymmetric and rounded, often reduced or absent on one side. Raphe strongly undulated with central pores small, round and close standing.

Habitat and distribution.-Pinnularia stidolphii was found in Traba and Ulla rivers. The Traba river is situated in a landscape dominated by granitic rocks. The study site was located at low altitude $-18 \mathrm{~m}$ a.s.1.at $14 \mathrm{~km}$ from the river source and a catchment area of $6.5 \mathrm{~km}^{2}$. The Ulla river runs through granites in contact with schists and basic rocks. The study site is also situated at low altitude $-64 \mathrm{~m}$ a.s.1.-, $96 \mathrm{~km}$ downstream of its source. Industrial activities and urban development are the main impacts, respectively. Probably abundant species in temperate regions of North and South Hemisphere, in waters poor in organic matter.

17. Planothidium daui (Foged) Lange-Bert., Iconogr. Diatomol. 6: 275 (1999); Achnanthes daui Foged, Danmark Geol. Undersoeg. 84: 14, pl. 1 fig. 10 (1962);
Achnantheiopsis daui (Foged) Lange-Bert., Arch. Protistenk. 148: 206 (1997). Fig. 4e.

Valves $7-12 \times 3.8-6 \mu \mathrm{m}$, elliptical or widely lanceolate in outline with clearly capitated ends. Striae 14-16 in $10 \mu \mathrm{m}$. The raphe valve has a narrow linear or lanceolate axial area generally with a distinct central area. Striae are strongly radiate on all sides, with striae at the centre more clearly shorter than the others. The rapheless valve is similar, but the straie are nearly parallel in the centre of the valve, changing to radiate near the poles.

Habitat and distribution.-The habitat is not clearly defined as a result of the problematic distinction from Planothidium granum (M.H.Hohn \& Hellerman) Lange-Bert. Uncommon in Galicia rivers and streams, relatively abundant $(>5 \%)$ at only one site, poor in organic content at $\mathrm{pH}$ close to neutral, with a rather low mineralization.

Note.-Often confused with Planothidium granum, but Planothidium daui has clearly beak-like to capitate apices and more linear valve edges.

18. Iconella delicatissima (F.W.Lewis) Ruck \& Nakov, Notul. Alg. 10: 3 (2016); Surirella delicatissima F.W.Lewis, Proc. Acad. Nat. Sci. Philadelphia 15: 343, fig. 4 (1864); Stenopterobia delicatissima (F.W.Lewis) Bréb. ex Van Heurck, Treat. Diat.: 374, figs. 19-51 (1896); Synedra acus var. delicatissima (F.W.Lewis) Grunow, Verh. K. K. Zool.Bot. Ges. Wien. 399: pl. 5/8, fig.15 (1862). Fig. 11f.

Valves $30-100 \times 3.5-9 \mu \mathrm{m}$, linear to linear-lanceolate with generally convex or parallel sides. Striae $18-27$ in $10 \mu \mathrm{m}$, clearly visible, interrupted in the middle by a narrow hyaline area. Frustules weakly silicified with narrow-linear isopolar longitudinal axis. Apices strongly narrowed, stretched and bluntly rounded. Easily distinguished of other related taxa by their sigmoid outline.

Habitat and distribution.-Cosmopolitan species but rare. Mostly observed sporadically in oligotrophic and dystrophic acids waters of siliceous mountain regions, well-oxygenated and low in organic matter and nutrients. Indicator of high ecological quality.

Note.-Based on phylogenetic analyses along with morphological studies Ruck \& al. (2016) have recently reclassified the Surirellales and proposed the transfer of Surirella delicatissima into a new monotypic genus, Iconella Jurilj.

19. Iconella linearis (W.Sm.) Ruck \& Nakov, Notul. Alg. 10: 2 (2016); Surirella linearis W.Sm., Syn. Brit. Diat. 1: 31, pl. 8, fig. 58 (1853); Suriraya linearis (W.Sm.) Pfitzer, Bot. Abh. (Bonn) 1(2): 112 (1871). Fig. 11j.

Valves $20-120 \times 9-25 \mu \mathrm{m}$, bilaterally symmetrical, isopolar, generally linear-lanceolate, lanceolate or even elliptical with cuneate or rounded poles; alar wings 2-3 in $10 \mu \mathrm{m}$. Striae 20-22 in $10 \mu \mathrm{m}$ fine and parallel at the center, becoming radiant ends, visible but not distinct. Pseudoraphe narrow and straight. The most obvious features of the valve are the corrugations of the surface associated with the formation of windows beneath the raphe system which give the valve a semblence of a series of transverse tubes opening under the raphe. 
Habitat and distribution.-Iconella linearis is considered a cosmopolitan species, common in slightly acidic to neutral $\mathrm{pH}$, weakly to moderately mineralized and moderately impacted by organic matter and nutrients.

Note.-Also transferred into genus Iconella by Ruck \& al. (2016) Very similar to Surirella roba Leclercq but Iconella linearis has a larger size and coarser structure, with more distantly spaced fibulae and corrugations.

\section{ACKNOWLEDGEMENTS}

The financial support for this work has been provided by Augas de Galicia (Xunta de Galicia) through a contract with the University of Santiago de Compostela for the assessment of the stream water quality in rivers of Galicia-Costa using diatom metrics (2005-2007). We are indebted to Juan Manuel Antelo Cortizas (Department of Química-Física) and Teresa García Bernadal (researcher of Santiago de Compostela University) for their assistance during fieldwork and insightful comments on stream chemistry and ecology. Special thanks are to Maria Penalta for the photomicrographs in this study. We also thank the reviewers for their very helpful and constructive comments. Finally, we also would like to acknowledge Dr. Alejandro Quintanar for the corrections and improvements made to the original manuscript.

\section{REFERENCES}

Aboal M., Alvárez Cobelas M., Cambra J. \& Ector L. 2003. Floristic list of the non marine diatoms (Bacillariophyceae) of Iberian Peninsula, Balearic Islands and Canary Islands. Update taxonomy and bibliography. In Witkowski A. (ed.), Diatom Monographs. A.R.G. Gantner Verlag K.G., Berlin.

Alles E., Nörpel-Schempp M. \& Langebertalot H. 1991. Zur Systematik und okologie charakteristischer Eunotia-Arten (Bacillariophyceae) in elektrolytarmen Bachoberlaufen. Nova Hedwigia 53: 171-213.

APHA 1998. Standard Methods for the Examination of Water and Wastewater ed. 20. American Public Health Association, American Water Works Association and Water Environment Association, Baltimore.

Bao R., Alonso A., Delgado C. \& Pagés J.L. 2007. Identification of the main driving mechanisms in the evolution of a small coastal wetland (Traba, Galicia, NW Spain) since its origin 5700 cal yr BP Palaeogeography, Palaeoclimatology, Palaeoecology 247: 296-312. https://doi.org/10.1016/j.palaeo.2006.10.019

Camburn K.E. \& Charles D.F. 2000. Diatoms of Low-Alkalinity Lakes in the Northeastern United States. Academy of Natural Sciences, Philadelphia.

CEN 2003. Water quality - Guidance standard for the routine sampling and pre-treatment of benthic diatoms from rivers for water quality assessment. EN 13946:2003. Comité European de Normalisation, Geneva.

CEN 2004. Water Quality-Guidance Standard for the Identification, Enumeration and Interpretation of Benthic Diatom Samples from Running Waters. EN 14407:2004. Comité European de Normalisation, Geneva.

Cleve P.T. 1895. Synopsis of the Naviculoid diatoms, Part II. Kongliga Svenska-Vetenskaps Akademiens Handlingar 27: 1-219.

Delgado C., Pardo I. \& García L. 2010. A multimetric diatom index to assess the ecological status of coastal Galician rivers (NW Spain). Hydrobiologia 644: 371-384. https://doi.org/10.1007/s10750-010-0206-y

Ector L. 1992. Control de la calidad biológica de las aguas superficiales en la red de aforos de Galicia-costa mediante diatomeas bénticas. In AnteloCortizas J.M. de, Calidad del agua en las estaciones de aforo de los ríos de Galicia. Años hidrológicos 1989-90, 1990-91: 76-157. Consellería de Ordenación do Territorio e Obras Públicas, Santiago de Compostela.
Gamundi J. 1911. Diatomeas de Santiago de Compostela y sus alrededores. Boletín de la Real Sociedad Española de Historia Natural 11: 388-394.

Guiry M.D. \& Guiry G.M. (eds.). 2015. AlgaeBase. World-wide electronic publication, National University of Ireland, Galway. Website: http://www. algaebase.org. [accessed November 2016].

Hofmann G., Werum M. \& Lange-Bertalot H. 2011. Diatomeen im Süßwasser-Benthos von Mitteleuropa. A.R.G. Gantner Verlag K.G., Ruggell, Liechtenstein.

Hustedt F. 1930. Bacillariophyta (Diatomeae). In Pascher A. (ed.), Die Süsswasser-Flora Mitteleuropas Heft 10. G. Fischer, Jena.

Juggins S. 2014. C2. Software for ecological and palaeoecological data analysis and visualisation. Version 1.7.5. Department of Geography, Newcastle.

Krammer K. 1997a. Die cymbelloiden Diatomeen. Eine Monographie der weltweit bekannten Taxa. Teil 1. Allgemeinen und Encyonema Part. In Lange-Bertalot H. (ed.), Biblioteca Diatomologica Band 36. J. Cramer, Berlin.

Krammer K. 1997b. Die cymbelloiden Diatomeen. Eine Monographie der weltweit bekannten Taxa. Teil 2. Encyonema Part., Encyonopsis und Cymbellopsis. In Lange-Bertalot H. (ed.), Biblioteca Diatomologica Band 37. J. Cramer, Berlin.

Krammer K. 2000. The genus Pinnularia. In Lange-Bertalot H. (ed.), Diatoms of the European Inland Waters and Comparable habitats. A.R.G. Gantner Verlag K.G., Koenigstein.

Krammer K. 2002. Cymbella. In Lange-Bertalot H. (ed.), Diatoms of the European Inland Waters and Comparable habitats. A.R.G. Gantner Verlag K.G., Koenigstein.

Krammer K. 2003. Cymbopleura, Delicata, Navicymbula, Gomphocymbellopsis, Afrocymbella. In Lange-Bertalot H. (ed.), Diatoms of Europe. Diatoms of the European Inland Waters and Comparable Habitats. A.R.G. Gärtner Verlag K.G., Koenigstein.

Krammer K. \& Lange-Bertalot H. 1985. Naviculaceae. Neue und wenig bekannte Taxa, neue Kombinationen und Synonyme sowie Bemerkungen zu einige Gattungen. In Lange-Bertalot H. (ed.), Biblioteca Diatomologica Band 9. J. Cramer, Berlin.

Krammer K. \& Lange-Bertalot H. 1988. Bacillariophyceae: Bacillariaceae, Epithemiaceae, Surirellaceae. In Ettl H., Gerloff J., Heynig H. \& Mollenhauer D. (eds.), Süßwasserflora von Mitteleuropa Band 2, Teil 2. G. Fisher Verlag, Stuttgart.

Krammer K. \& Lange-Bertalot H. 1991a. Bacillariophyceae: Centrales, Fragilariaceae, Eunotiaceae. In Ettl H., Gerloff J., Heynig H. \& Mollenhauer D. (eds.), Süßwasserflora von Mitteleuropa Band 2, Teil 3. G. Fisher Verlag, Stuttgart.

Krammer K. \& Lange-Bertalot H. 1991b. Bacillariophyceae: Achnanthaceae, Kritische Ergäzungen zu Navicula (Lineolatae) und Gomphonema. In Ettl H., Gärtner G., Gerloff J., Heynig H. \& Mollenhauer D. (eds.), Süßwasserflora von Mitteleuropa Band 2, Teil 4. G. Fisher Verlag, Stuttgart.

Lange-Bertalot H. 1999. Neue Kombinationen von Taxa aus Achnanthes Bory (sensu lato). In Lange-Bertalot H. (ed.), Iconographia Diatomologica. [Annotated Diatom Micrographs vol. 6.] Koeltz Scientific Books, Königstein.

Lange-Bertalot H. 2001. Navicula sensu stricto. 10 Genera separated from Navicula sensu lato Frustulia. In Lange-Bertalot H. (ed.), Diatoms of the European Inland Waters and Comparable habitats. A.R.G. Gantner Verlag K.G., Koenigstein.

Lange-Bertalot H. \& Krammer K. 1989. Achnanthes, eine Monographie der Gattung. In Lange-Bertalot H. (ed.), Bibliotheca Diatomologica 18. J. Cramer, Berlin. 
Lange-Bertalot H. \& Metzeltin D. 1996. Indicators of oligotrophy. 800 taxa representative of three ecologically distinct lake types: carbonate buffered, oligodystrophic, weakly buffered soft water. In Lange-Bertalot H. (ed.), Iconographia Diatomologica 2. Koeltz, Königstein.

Lange-Bertalot H., Bak M., Witkowski A. \& Tagliaventi N. 2011. Eunotia and some related genera. In Lange-Bertalot H. (ed.), Diatoms of Europe. Diatoms of the European Inland Water and Comparable Habitats. A.R.G. Gantner, Ruggell, Liechtenstein.

López-Rodríguez M.C. \& Penalta-Rodríguez M. 2007. Freshwater algae in Galician Central Macizo rivers (NW Spain) with new records for Iberian Peninsula. Algological Studies 125: 57-77. https://doi.org/10.1127/1864-1318/11/0125-057

Lowe R.L., Kociolek P., Johansen J.R., Van de Vijver B., Lange-Bertalot H. \& Kopalová K. 2014. Humidophila gen.nov., a new genus for a group of diatoms (Bacillariophyta) formerly within the genus Diadesmis: species from Hawai'i, including one new species. Diatom Research 29: 351-360.

Margalef R. 1955. Comunidades bióticas de las aguas dulces del noroeste de España. Publicaciones del Instituto de Biología Aplicada 21: 5-85.

Margalef R. 1956. Algas de agua dulce del norte de España. Publicaciones del Instituto de Biología Aplicada 22: 5-47.

Moser G., Lange-Bertalot H. \& Metzeltin D. 1998. Insel der Endemiten Geobotanisches Phänomen Neukaledonien (Island of endemics New Caledonia - a geobotanical phenomenon). Bibliotheca Diatomologica 38: $1-464$.

Ortiz-Lerín R. \& Cambra J. 2007. Distribution and taxonomic notes on Eunotia Ehrenberg 1837 (Bacillariophyceae) in rivers and streams of Northern Spain. Limnetica 26: 415-434.

Patrick R.M. \& Reimer C.W. 1966. The diatoms of the United States exclusive of Alaska and Hawaii (vol. 1). Monographs of the Academy of Natural Sciences of Philadelphia 13: 1-688.
Penalta-Rodríguez M. \& López-Rodríguez M.C. 2006. Contribution à la flore des diatomées épilithiques du Massif Central Galicien (Ourense, Espagne). Simbioses 14: 21-25.

Penalta-Rodríguez M. \& López-Rodríguez M.C. 2007. Diatomeas y calidad del agua de los ríos del Macizo Central Gallego (Ourense, N.O. España) mediante la aplicación de índices diatomológicos. Limnetica 26: 351-358.

Reichardt E. 1999. Zur Revision der Gattung Gomphonema. Die Arten um G. affine/insigne, G. angustatum/micropus, G. acuminatum sowie gomphonemoide Diatomeen aus den Oberoligozän in Böhmen. In LangeBertalot H. (ed.), Iconographia Diatomologica 8. Koeltz Scientifc Books, Berlin.

Round F.E., Crawford R.M. \& Mann D.G. 1990. The diatoms biology and morphology of the genera. Cambridge University. Cambridge.

Ruck E.C., Nakov T., Alverson A.J. \& Theriot E.C. 2016. Nomenclatural transfers associated with the phylogenetic reclassification of the Surirellales and Rhopalodiales. Notulae Algarum 10: 1-4.

Van Dam H., Mertens A., \& Sinkeldam J. 1994. A coded checklist and ecological indicator values of freshwater diatoms from The Netherlands. Netherlands Journal of Aquatic Ecology 28: 117-133. https://doi.org/10.1007/BF02334251

Varela M. 1976. Diatomeas de Santiago de Compostela y alrededores. PhD Thesis, Universidad de Santiago de Compostela, Santiago de Compostela.

Varela M. 1982. Adiciones a la flora de diatomeas de agua dulce de Galicia. Collectanea botanica 13: 977-985.

Varela M., Rodríguez B. \& Costas E. 1992. Inventario de diatomeas de auga doce de Galicia. Inventarios do Seminario de Estudos Galegos 9: $11-55$. 